\title{
Nematic-nematic phase separation in binary mixtures of thick and thin hard rods: Results from Onsager-like theories
}

\author{
Szabolcs Varga, ${ }^{1, *}$ Kirstin Purdy, ${ }^{2}$ Amparo Galindo,${ }^{3}$ Seth Fraden, ${ }^{2}$ and George Jackson ${ }^{3}$ \\ ${ }^{1}$ Department of Physics, University of Veszprém, H-8201 Veszprém, P.O. Box 158, Hungary \\ ${ }^{2}$ Martin Fisher School of Physics, Brandeis University, Waltham, Massachusetts 02454, USA \\ ${ }^{3}$ Department of Chemical Engineering, Imperial College London, South Kensington Campus, London SW7 2AZ, United Kingdom
}

(Received 17 May 2005; published 7 November 2005)

\begin{abstract}
The fundamental nature of the nematic-nematic $(N-N)$ phase separation in binary mixtures of rigid hard rods is analyzed within the Onsager second-virial theory and the extension of Parsons and Lee which includes a treatment of the higher-body contributions. The particles of each component are modeled as hard spherocylinders of different diameter $\left(D_{1} \neq D_{2}\right)$, but equal length $\left(L_{1}=L_{2}=L\right)$. In the case of a system which is restricted to be fully aligned (parallel rods), we provide an analytical solution for the spinodal boundary for the limit of stability of $N-N$ demixing; only a single region of $N-N$ coexistence bounded at lower pressures (densities) by a $N-N$ critical point is possible for such a system. The full numerical solution with the Parsons-Lee extension also indicates that, depending on the length of the particles, there is a range of values of the diameter ratio $\left(d=D_{2} / D_{1}\right)$ where the $N-N$ phase coexistence is closed off by a critical point at lower pressure. A second region of $N-N$ coexistence can be found at even lower pressures for certain values of the parameters; this region is bounded by an "upper" $N-N$ critical point. The two $N-N$ coexistence regions can also merge to give a single region of $N-N$ coexistence extending to very high pressure without a critical point. By including the higher-order contributions to the excluded volume (end effects) in the Onsager theory, we prove analytically that the existence of the $N-N$ lower critical point is a direct consequence of the finite size of the particles. A new analytical equation of state is derived for the nematic phase using the Gaussian approximation. In the case of Onsager limit (infinite aspect ratio), we show that the $N-N$ phase behavior obtained using the Parsons-Lee approach substantially deviates from that with the Onsager theory for the $N-N$ transition due to the nonvanishing third and higher order virial coefficients. We also provide a detailed discussion of the $N-N$ phase behavior of recent experimental results for mixtures of thin and thick rods of the same length, for which the Onsager and Parsons-Lee theories can provide a qualitative description.
\end{abstract}

DOI: 10.1103/PhysRevE.72.051704

PACS number(s): 64.70.Md, 64.60.Cn, 61.30.Cz, 61.30.St

\section{INTRODUCTION}

It is well-established that rodlike molecules can give rise to liquid crystalline phases [1]. The transition between an isotropic and a nematic phase was first explained by Onsager [2] in terms of competing orientational and translational entropies in a fluid of infinitely long and thin hard particles, a result that suggested the molecular alignment in these systems was dominated by molecular shape rather than by specific attractive interactions. Flory [3] reached a similar conclusion using a lattice approach. Some 30 years later this view was confirmed by computer simulation [e.g., isotropic $(I)$, nematic $(N)$, and smectic $A(\operatorname{Sm} A)$ phases can be stabilized in a system of hard spherocylinders [4]], and several experimental systems have now also been designed which support the existence of liquid crystalline phases in fluids of purely repulsive monodisperse $[5,6]$ and polydisperse rodlike particles (e.g., see [7]).

In his seminal paper of 1949 , Onsager already points at the interesting phase behavior that must arise in mixtures of anisotropic particles. He suggested that a strong fractionation will occur in a mixture of rodlike particles of different lengths, with a higher proportion of the longer particles in

*Corresponding author. Email address: vargasz@almos.vein.hu the more ordered phase. Onsager did not, however, report any calculations for mixtures in his paper. The first confirmation of such an effect is due to Flory and Abe [8], who calculated the phase diagram of a mixture of two monodisperse semiflexible polymers of different aspect ratio and obtained the strong fractionation predicted by Onsager, and in addition reported a regions of nematic-nematic $(N-N)$ and isotropic-nematic-nematic $(I-N-N)$ coexistence. Experimental support for the calculations of Abe and Flory was provided by Itou and Teramoto $[9,10]$ using two wellfractionated polysaccharides (with aspect ratios of 18.3 and 223) in aqueous solution. They were able to observe a strong fractionation of long and short particles, and a region of $I-N-N$ coexistence. Itou and Teramoto did not investigate high-density solutions corresponding to low water content, and so the $N-N$ region expected at higher density was not reported in their work. There is now, however, ample experimental evidence of mixtures exhibiting $N-I-N$ demixing: Buining and Lekkerkerker [11] have reported $N-N$ coexistence in a polydisperse system of rodlike colloids, and shortly afterwards Nouensis $e t$ al. [12] observed such a transition in a binary mixture of uniaxial nematic liquid crystals. Large regions of $N-N$ demixing can also be observed in binary mixtures of rodlike and platelike colloidal particles $[13,14]$.

Very recently, we have reported experimental evidence for $N-N$ demixing in a binary mixture monodisperse "purely re- 
pulsive" rodlike particles of equal length and different diameter [15]. One of the components is a charged semiflexible $f d$ virus [5], and the other the $f d$ virus irreversibly coated with the neutral polymer polyethylene glycol (PEG) [16], both dispersed in aqueous solution. In these systems the particles of the two components can be considered to be of the same length but of different diameter. The bare $f d$ virus is thus the thin rods and the PEG-coated virus the thick rods. The effective diameter of the bare virus can be tuned by changing the ionic strength of the solution, which allows for the investigation of mixtures of varying particle diameters, and an exhaustive study of the types of behavior possible in such systems. We found isotropic-nematic-nematic coexistence as well as $N-N$ demixing, as seen in previous studies [11-14]. In contrast, however, by examining the phase diagram well into the denser nematic region, a previously unobserved lower nematic critical point was found for particular values of the diameter ratio (cf. Fig. 1).This is of special interest not just from a practical or experimental point of view, but also from a theoretical point of view, as the nature and types of nematic-nematic demixing transition in these systems have been a point of debate for sometime.

Nematic-nematic separation has been reported in surprisingly few simulation studies; the work of Camp et al. [17] appears to be the only such study. They used the Gibbs ensemble Monte Carlo technique to calculate the phase diagram of a binary mixture of hard uniaxal ellipsoids, one rodlike and the other platelike, and observed a marked nematicnematic separation. The phase behavior of mixtures of prolate and oblate particles is further complicated by the promise of biaxiality, and is thus not directly relevant to our current study. Binary mixtures of rodlike and platelike particles of aspect ratios closer to those of thermotropic liquid crystals have also been considered by simulation, but in this case nematic-columnar, and smectic $A$-columnar coexistence are observed $[18,19]$. An induced alignment of the short particles in a phase rich in longer ones is reported in a recent study of binary mixtures of hard Gaussian overlap particles of different aspect ratio, but no phase separation was found [20]. Isotropic-isotropic demixing bounded by a lower critical point has been found by Bolhuis et al. [21] from Gibbs ensemble Monte Carlo simulations for a system of spherocylinders with attractive interactions, mimicking a colloidpolymer mixture. In the same work a $N-N$ demixing transition is predicted for the Onsager limit, though no simulations were carried in this limit. Similar results were obtained by Dijkstra and van Roij [22] in a binary mixture of hard spherocylinders of equal lengths and varying diameters. This study is of particular relevance to us since our experimental system also corresponds to a mixture of rodlike particles of equal length but different diameters. Dijkstra and van Roij simulated regions of isotropic-isotropic and isotropicnematic coexistence, but did not investigate the highpressure states where nematic demixing is possible.

On the other hand, nematic-nematic coexistence has been predicted by several theoretical approaches. As mentioned earlier, this type of demixing was first reported by Flory and Abe in 1978 [8] using a lattice model of bidisperse hard, rodlike polymers. Since then there have been a number of predictions supporting this finding: Birshtein et al. [23] used
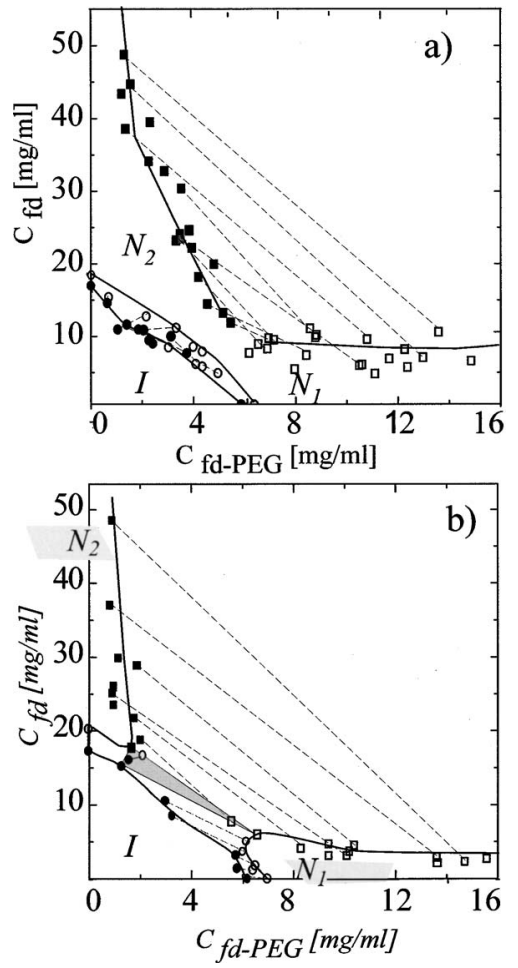

FIG. 1. Experimental phase diagram of a suspension of a mixture of charged semiflexible $f d$ virus (thin rods) and $f d$ virus coated with poly(ethylene glycol), $f d$-PEG (thick rods) of the same length but different diameter. The phase diagrams are presented in a concentration-concentration plane for the following molecular parameters (aspect ratio $k=L_{\text {thick }} / D_{\text {thick }}$ and diameter ratio $d$ $=D_{\text {thin }} / D_{\text {thick }}$ ) and conditions ( $p \mathrm{H}$ and ionic strength): (a) $k=24, d$ $=0.345, p \mathrm{H}=8.2$ and $I=50 \mathrm{mM}$; (b) $k=24, d=0.333, p H=8.2$ and $I=60 \mathrm{mM}$. The continuous curves are a guide to the eye and represent the phase boundaries of the isotropic-nematic, and nematicnematic coexistence regions, while the dashed lines are the tie lines connecting the coexisting phases. The circles and squares denote the experimentally obtained phase boundaries along the $I-N$ and $N-N$ phase transitions, respectively. The labels $I, N_{1}$, and $N_{2}$ denote the isotropic, the $f d$-PEG rich nematic, and the $f d$-rich nematic phases, respectively. The three-phase region is indicated by the gray triangle.

an extension of the theory of Onsager to mixtures proposed by Lekkerkerker et al. [24], and found that $N-N$ separation is possible when the ratio of the lengths of the two components exceeds a certain value. Vroege and Lekkerkerker [25] estimated this ratio to be 3.167 using a Gaussian trial function method, and a calculation with an exact method to compute the high-density orientational distribution functions confirmed their conclusions [26]; it should be noted that a later result using a fully numerical technique placed the value of the length ratio at 2.91 [27]. The general case of $N-N$ demixing in binary mixtures of hard, rodlike particles of arbitrary size (i.e., with differing diameters and lengths) was considered by Hemmer [28], who mapped the parameterspace where the transition is predicted with the Onsager approach and a Gaussian trial function for the equilibrium distribution functions. The demixing of the nematic phase in mixtures of hard, rodlike particles has also been studied (to different lev- 
els of approximation within the Onsager approach) by Sear and Mulder [29], van Roij and co-workers [30,31], Speranza and Sollich [32], and very recently by some of us [15,33]. It is also worth mentioning that $N-N$ demixing can also be predicted with a Maier-Saupe approach [34,35], but in this case it is the effective attractive interactions that play a major role.

An interesting unresolved point that has arisen from this body of literature relates to the origin and nature of the nematic demixing transition. In their original phase diagram, Flory and Abe [8] presented a demixed region bounded by a critical point at high density. As Flory and Abe studied an athermal system within an incompressible lattice theory, the dependence on pressure or temperature is not relevant in their case, and the critical point can be presented in composition space. Interestingly, in their early calculations Vroege and Lekkekerker [25] did not present a critical point; instead, a three-phase point is found from which the nematic-nematic separation continues to high pressures. Speranza and Sollich [36] have recently reported $N-N$ coexistence in a system of length-bidisperse rods; they conclude that in the range of length ratios between 2.91 (cf. the paper by Varga and Szalai [33]) and 3.2 the phase diagram exhibits $N-N$ demixing ending at a high density (pressure) critical point. Using the Onsager theory, van Roij et al. [31] present a systematic study of the phase behavior for varying diameter ratio, reconciling the different types of phase diagram predicted for binary mixtures of thick and thin rods. Above a certain value of the diameter ratio, they observe the appearance of a region of $N-N$ demixing, bounded by an $I-N-N$ three-phase line at lower density (pressure) and by an upper critical point at higher density (pressure). As the diameter ratio is further increased, the $N-N$ demixing is seen to dominate the phase diagram and it continues from the $I-N-N$ triple point to very high pressures, never ending at a critical point (as seen with the previous calculations of Vroege and Lekkerkerker [25]). For very large diameter ratios $I-I$ demixing is found.

Earlier, van Roij and Mulder [30] had also suggested that a region of $N-N$ demixing bounded at lower pressure by a critical point is possible in a system of parallel hard rods. While $N-N$ demixing had been previously explained as driven by the orientational entropy and not by the exclude volume, clearly this cannot be the case in a parallel system, and hence they interpret the demixing as a depletion-driven transition. A formal proof of the existence of such a critical point was later provided by Perera $[37,38]$ within a density functional approach for different types of hard-body systems. A phase diagram exhibiting a $N-N$ demixed region with a lower critical pressure has been predicted by Wensik et al. [39] in a mixture of thin and thick plates. It has been predicted as well as observed in mixtures of rodlike particles in our recent work $[15,33]$. A number of aspects that are important to consider for an accurate description of the $N-N$ demixing have previously been highlighted $[40,41]$. These include: the incorporation of the usually neglected "end effects" [40] (which arise from the finite size of the particles); taking into account virial coefficients higher than the second (with, e.g., a scaling approach); and the accuracy of the self-consistent calculations for the orientational distribution functions [41]. In this paper we look at these issues in some detail. The focus is on isotropic and uniaxial nematic phases, and we do not attempt to incorporate more ordered phases such as biaxial nematics, smectics, columnars, or solids, though it is clear that they will certainly be the stable phases relative to the nematic states at high pressures (densities).

This paper is organized as follows: binary mixtures of parallel rods are examined using a virial expansion of the free energy at the level of the second virial coefficient in the following section; analytical formulas are derived for the spinodal decomposition (limit of material stability) and for the critical point of a mixture of thick and thin rods. In the limit of zero diameter ratio, the critical properties are given exactly. In Sec. III we give a short description of the Onsager- (Sec. III A) and Parsons-Lee theories (Sec. III B) for the nematic phase. By applying the trial function method for the minimization of the free-energy functional, we show that analytical equations can be derived for the pressure of the highly ordered nematic phases. A binodal analysis (coexistence) of the possible demixing transitions is presented and a comparison is made between the Onsager and Parsons-Lee theories in mixtures of very long thick and thin rods (Onsager limit). In Sec. III C we discuss the reliability of the trial function method with Onsager's original trial function, and the shortcomings of the Gaussian approximation is highlighted. On the basis of Parsons-Lee theory, a classification of the phase diagrams and global phase behavior, including the isotropic and nematic phases only, is presented in Sec. IV. Finally, we make some general conclusions in Sec. V.

\section{ONSAGER THEORY FOR A MIXTURE OF THICK AND THIN PARALLEL HARD RODS}

In this section, we consider a binary mixture of parallel hard rods, modeled as hard spherocylinders of equal lengths $\left(L_{1}=L_{2}=L\right)$, but of different diameters $\left(D_{1} \neq D_{2}\right)$, and search for the molecular conditions for demixing, i.e., a phase separation between two phases of the same symmetry (nematicnematic or isotropic-isotropic). We assume that the particles are very long and thin, so that the Onsager limit is satisfied; for convenience, we use a free-energy density (as opposed to the free energy per particle), and write it as the sum of ideal and residual contributions

$$
a=\frac{A}{k_{B} T V}=a_{i d}+a_{\text {res }},
$$

where $T$ is the temperature, $k_{B}$ Boltzmann's constant, and $V$ the volume of the system. The ideal term of a binary mixture is given analytically in terms of number densities of the components $\rho_{i}=N_{i} / V(i=1,2)$ as

$$
a_{i d}=\sum_{i=1}^{2} \rho_{i} \ln \rho_{i}-\rho_{i}=\rho_{1} \ln \rho_{1}-\rho_{1}+\rho_{2} \ln \rho_{2}-\rho_{2} .
$$

Note that the de Broglie kinetic contributions do not affect the phase behavior and are omitted from the expression for compactness. Following the seminal work of Onsager [2], the residual contribution to the free energy can be written 
using an approximate virial theory truncated at the level of the second virial coefficients $\left(B_{2}^{i j}\right)$, so that

$$
a_{\text {res }}=\sum_{i, j=1}^{2} B_{2}^{i j} \rho_{i} \rho_{j},
$$

where the second virial coefficient $B_{2}^{i j}$ between two particles of components $i$ and $j$ is half of their pair excluded volume $\left(v_{e x c}^{i j}\right)$. In the special case of a parallel system, where the long symmetry axes of all particles are constrained along a given direction, the second virial coefficients of hard spherocylinders are easily determined as

$$
B_{2}^{i j}=\frac{1}{2} \nu_{e x c}^{i j}=\frac{\pi}{12}\left(D_{i}+D_{j}\right)^{3}+\frac{\pi}{4}\left(D_{i}+D_{j}\right)^{2} L .
$$

Using Eqs. (2)-(4) the free-energy density of the system of thick and thin parallel rods in the special limit of very long and thin particles (the Onsager limit) is obtained as

$$
a^{*}=\frac{A \nu_{1}}{k_{B} T V}=\sum_{i=1}^{2} c_{i}\left(\ln c_{i}-1\right)+4 c_{1}^{2}+2(1+d)^{2} c_{1} c_{2}+4 d^{2} c_{2}^{2},
$$

where the last three terms correspond to the residual free energy. Note that for the sake of brevity and for other practical reasons Eq. (5) is written in dimensionless form, where $c_{i}=\rho_{i} \nu_{1}$ is the reduced number density of component $i, d$ $=D_{2} / D_{1}$ is the diameter ratio and $v_{1}=(\pi / 4) L D_{1}^{2}$ is the volume of a cylinder of length $(L)$ and the diameter $\left(D_{1}\right)$. It is interesting to note from Eq. (5) that, as we have assumed the Onsager limit, only the terms representing the cylindrical part of the particle appear, while the lower order terms representing the spherical caps make no contribution. This means that there is no difference between a cylinder and a spherocylinder model in the Onsager limit in the case of a parallel system; the spherical caps play a role only when the particle length and diameter are of the same order of magnitude.

In order to determine the binodal curve corresponding to the equilibrium phase separation of the mixture, the chemical potentials of each components and the overall pressure are required. In our system these are easily obtained from the reduced free-energy density [Eq. (5)] as

$$
\begin{gathered}
\mu_{1}^{*}=\left(\frac{\partial a^{*}}{\partial c_{1}}\right)_{c_{2}}=\ln c_{1}+8 c_{1}+2(1+d)^{2} c_{2}, \\
\mu_{2}^{*}=\left(\frac{\partial a^{*}}{\partial c_{2}}\right)_{c_{1}}=\ln c_{2}+2(1+d)^{2} c_{1}+8 d^{2} c_{2}, \\
P^{*}=-a^{*}+\sum_{i=1}^{2} c_{i} \mu_{i}^{*}=c_{1}+c_{2}+4 c_{1}^{2}+2(1+d)^{2} c_{1} c_{2}+4 d^{2} c_{2}^{2},
\end{gathered}
$$

where the dimensionless quantities have been defined as $\mu_{i}^{*}$ $=\mu_{i} / k T$ and $P^{*}=P \nu_{1} / k T$. The determination of the binodal curve requires solving for the equality of pressure and chemical potentials of each of the components in the coexisting phases, here denoted as phases $I$ and $I I: P_{I}=P_{I I}, \mu_{1 I}$ $=\mu_{1 I I}$, and $\mu_{2 I}=\mu_{2 I I}$. Finding the solution to these conditions can be rather cumbersome; even with advanced numerical techniques a good estimate of an initial point is usually required. Instead of a random search for the phase equilibria, we first carry out a stability analysis for the limit of material stability of the mixture and calculate the so-called spinodal [42], to determine the range of diameter ratios where phase instabilities may take place on mixing of the two components. An important advantage of this condition is that it can be expressed in terms of free-energy density in a very compact form [42] as

$$
\frac{\partial^{2} a^{*}}{\partial c_{1}^{2}} \frac{\partial^{2} a^{*}}{\partial c_{2}^{2}}-\left(\frac{\partial^{2} a^{*}}{\partial c_{1} \partial c_{2}}\right)^{2}=0 .
$$

For a mixture of thick and thin parallel hard rods, the substitution of the density derivatives of the free energy given in Eq. (5) into Eq. (8) gives our working equation for the spinodal as

$$
c_{1}=\frac{1}{4} \frac{1+8 d^{2} c_{2}}{\left\{(1+d)^{4}-16 d^{2}\right\} c_{2}-2} .
$$

This equation can be rewritten in terms of the total density $\left(c=c_{1}+c_{2}\right)$ and composition $\left(x_{i}=N_{i} / N\right)$ as $c_{1}=c x_{1}$ and $c_{2}$ $=c x_{2}$; in practice, we solve Eq. (9) for $c$ at a given diameter ratio $d$ and mole fraction of component 2 . We also choose component 1 to be that with the largest diameter, i.e., $d<1$. It is important to note that Eq. (9) has no physical solution in the limits of $d=1$ and of the pure phases of component 1 and $2(x=0$ or 1$)$. In the first limit $(d=1) c_{1}$ is negative, while in the pure phase limits either $c_{1}<0$ or $c_{2}<0$. Another important property of Eq. (9) is that $c_{1}$ is positive (negative) for $c_{2}$ higher (less) than $2 /\left\{(1+d)^{4}-16 d^{2}\right\}$, while $c_{1}$ is undefined when $c_{2}=2 /\left\{(1+d)^{4}-16 d^{2}\right\}$. Since both reduced densities have to be positive, only one of the two resulting curves of Eq. (9) can be the spinodal of the system. This also means that the system of parallel hard rods can only have one critical point and $N-N$ coexistence region.

In addition to the condition of stability given in Eq. (8), the critical point of the binary mixture can be determined by ensuring that the third derivative of the Gibbs free energy with respect to the mole fraction also vanishes [42]. This can be rewritten in terms of the reduced Helmholtz free-energy density as

$$
\begin{gathered}
\frac{\partial^{3} a^{*}}{\partial c_{1}^{3}}-3 \frac{\frac{\partial^{2} a^{*}}{\partial c_{1} \partial c_{2}}}{\frac{\partial^{2} a^{*}}{\partial c_{2}^{2}}} \frac{\partial^{3} a^{*}}{\partial c_{1}^{2} \partial c_{2}}+3\left(\frac{\frac{\partial^{2} a^{*}}{\partial c_{1} \partial c_{2}}}{\frac{\partial^{2} a^{*}}{\partial c_{2}^{2}}}\right)^{2} \frac{\partial^{3} a^{*}}{\partial c_{1} \partial c_{2}^{2}} \\
-\left(\frac{\frac{\partial^{2} a^{*}}{\partial c_{1} \partial c_{2}}}{\frac{\partial^{2} a^{*}}{\partial c_{2}^{2}}}\right)^{3} \frac{\partial^{3} a^{*}}{\partial c_{2}^{3}}=0 .
\end{gathered}
$$

An unexpectedly compact expression is obtained for the 


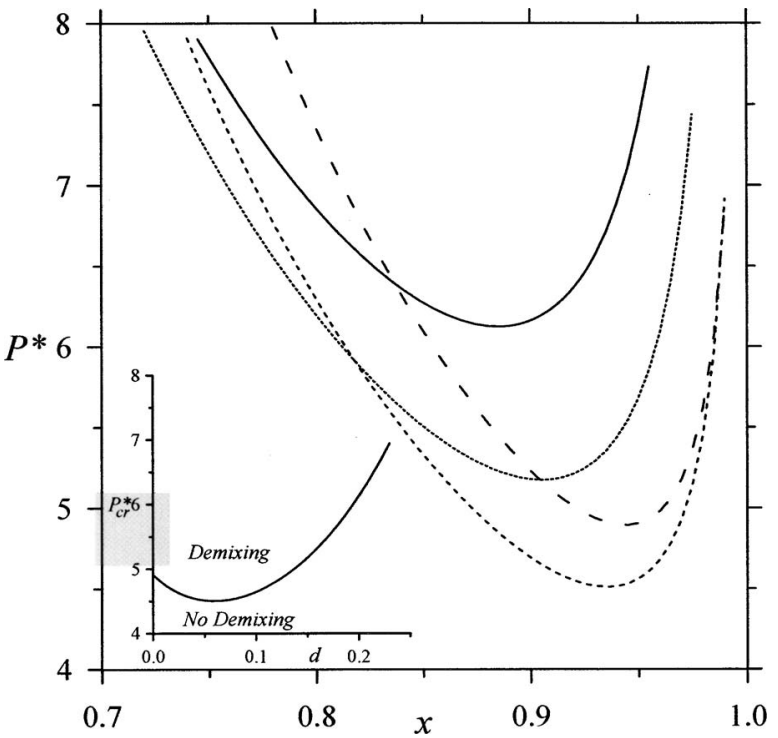

FIG. 2. Reduced pressure-composition $\left(P^{*}-x\right)$ representation of the spinodal decomposition of a binary mixture of parallel thick (component 1) and thin (component 2) hard rods for various diameter ratios $d=D_{2} / D_{1}: d=0.2$ (solid), 0.15 (dotted), 0.05 (shortdashed) 0.001 (long-dashed). In the inset we show the reduced pressure $\left(P_{c r}^{*}\right)$ as a function of diameter ratio at lower critical point of the demixing transition. The mole fraction is that of the thin rods $\left(x=x_{2}\right)$.

critical point in the case of a mixture of thick and thin parallel hard rods

$$
c_{1}^{2}=\frac{\left(1 / c_{2}+8 d^{2}\right)^{3} c_{2}^{2}}{8(1+d)^{6}} .
$$

The simultaneous solution of Eqs. (9) and (11) gives the critical reduced densities $\left(c_{1, c r}, c_{2, c r}\right)$ of the demixing transition at a given diameter ratio. The total reduced density and the compositions can then be obtained using $c=c_{1}+c_{2}$ and $x_{i}=c_{i} / c$.

In Fig. 2 the spinodal boundaries of a number of selected systems are presented in the pressure-composition $P^{*}-x$ plane. Each of the curves was obtained substituting the solution of Eq. (9) into the expression of the reduced pressure given in Eq. (7). The most important feature of Fig. 2 is that for decreasing diameter ratios up to $d \approx 0.06$ an enhancement of the phase separation region (lowering of the lower critical point) is observed. A further decrease in $d$, however, shifts the spinodal curves in the direction of higher pressures, i.e., the mixed state is stable with respect to demixing. This effect can be seen more clearly in the inset of the figure, where the lower critical points are presented as a function of the diameter ratio $d$. This special behavior is due to the subtle competition between the excluded volume interactions between the unlike components and those of the thin component, as only these two interactions can be changed with varying diameter ratio. On decreasing the diameter ratio from $d=1$ to 0 , both $B_{2}^{22}$ and $B_{2}^{12}$ decrease from $4 \nu_{1}$ to their limiting values of $B_{2}^{22}=0$ and $B_{2}^{12}=\nu_{1}$. At $d \approx 0.06, B_{2}^{22}$ is virtually equal to zero, while $B_{2}^{12}$ is still considerably different from its limiting value $\left(B_{2}^{12}=\nu_{1}\right)$. As a result, the decreasing unlike excluded volume characterized by $B_{2}^{12}$ does not promote the demixing transition in the interval of $0<d<0.06$. It is also interesting to note that the system still demixes at $d=0$, with an associated critical point at $c_{1, c r}=1 /(1+\sqrt{17}), c_{2, c r}=(9+\sqrt{17}) / 4$, and $P_{c r}^{*}=(67+15 \sqrt{17}) /(1+\sqrt{17})^{2}$. These critical values can be obtained analytically because Eqs. (9) and (11) become very simple in the limit of $d=0$. The subtle diameter-ratio dependence of the critical pressure and the spinodal curves indicates that the shape and size difference between the particles of the two components is necessary but not sufficient for the phase separation, because the mixed state becomes more stable for not only high values of diameter ratios but also for small values. It is also important to point out that only a single region of $N-N$ coexistence bounded by a critical point at lower pressure (density) is seen for the system of parallel hard rods of equal length and different diameter; as we will see later, an additional region of $N-N$ demixing bounded by an upper-critical pressure can be found in the case of the freely rotating system. Finally, we should emphasize that the demixing transition in binary mixture of parallel hard rods has already been studied by van Roij and Mulder [30] and DuBois and Perera [37]. Working in the context of a geometrical density functional theory, DuBois and Perera [37] found that some special systems may exhibit closed-loop immiscibility, where the phase coexistence curve is bounded by upper and lower critical points. The mixture of parallel thick and thin rods was not, however, considered in their study.

In summary, our principal finding for the system of parallel rods of equal length but different diameter is that only a single $N-N$ demixing region is found for a range of values of the diameter ratio; the region is bounded at lower pressures by a critical point.

\section{ONSAGER AND PARSONS-LEE THEORIES FOR A MIXTURE OF FREELY ROTATING THICK AND THIN HARD RODS}

Model systems of parallel particles are very useful for simple and accurate theoretical studies, but are not, unfortunately, easily attainable experimentally due to the rotational degrees of freedom of real particles. In this section we extend our study to the general case when the orientations of the rods are not restricted and can, in principle, point in any direction of space. We use two well-established approaches, the second-virial theory of Onsager [2] (Sec. III A), and the Parsons-Lee (PL) $[43,44]$ scaling of the Onsager approach to incorporate higher virial coefficients (Sec. III B). Onsager [2] devised his theory for an accurate description of the $I-N$ phase transition in a model system of long hard rods, while Parsons and Lee provided an approach that can be considered as a generalization of Onsager's theory to maintain accuracy in systems of intermediate and short particles. Although it may be naturally expected for the PL theory to converge to the Onsager solution in the limit of $L / D \rightarrow \infty$, we find this is not always the case. Here, we show that for mixtures of rods the PL theory substantially deviates from that of Onsager in the nematic phase, even in the limit of $L / D$ $\rightarrow \infty$. In order to get analytical results, we use the trial func- 
tion originally proposed by Onsager [2] for the representation of the single-particle orientational distribution functions in the fluid. In seeking a level of simplicity in the solution of the coexistence conditions, a number of further approximations have also been used; we devote Sec. III C to discuss the effect of the simplifying assumptions.

\section{A. The Onsager theory}

The free energy of a mixture of hard rods with unrestricted degrees of rotational freedom can be written following the form of Eq. (1) in terms of the Helmholtz free-energy density. The crucial difference is that the free energy in this general case is a functional of the orientational distribution functions (ODF) of the components of the mixture. Each ODF gives the probability of finding a particle with a given orientation, and depends on the unit vector $\underline{\omega}$ describing the orientation of the particles of a given component.

The ideal contribution of the free-energy density contains a term proportional to the entropy of mixing, as before [see Eq. (2)], and a term proportional to the orientational entropy, so that

$$
a_{i d}=\rho_{1} \ln \rho_{1}-\rho_{1}+\rho_{2} \ln \rho_{2}-\rho_{2}+\rho_{1} \sigma\left[f_{1}\right]+\rho_{2} \sigma\left[f_{2}\right],
$$

where $f_{1}$ and $f_{2}$ are the ODF of components 1 and 2, respectively. The $\sigma$ functional introduced by Onsager [2] is given as

$$
\sigma\left[f_{i}\right]=\int f_{i}(\underline{\omega}) \ln \left(4 \pi f_{i}(\underline{\omega})\right) d \underline{\omega} \quad(i=1,2) .
$$

Note that this functional is at a minimum for the case of an isotropic distribution, where $\sigma\left[f_{i}=1 / 4 \pi\right]=0$, hence favoring orientationally disordered states.

The form of the residual free energy can also be written following Eq. (3), but the second virial coefficients are now functionals of the ODF, written in terms of the pair excluded volume as

$$
B_{2}^{i j}=\frac{1}{2} \iint \nu_{e x c}^{i j}\left(\underline{\omega}_{1} \underline{\omega}_{2}\right) f_{i}\left(\underline{\omega}_{1}\right) f_{j}\left(\underline{\omega}_{2}\right) d \underline{\omega}_{1} d \underline{\omega}_{2} .
$$

The excluded volume between two hard spherocylinders $i$ and $j$ of different diameter $D_{i}$ and $D_{j}$ but equal length $L$ is given by

$$
\nu_{e x c}^{i j}(\gamma)=\frac{\pi}{6}\left(D_{i}+D_{j}\right)^{3}+\frac{\pi}{2}\left(D_{i}+D_{j}\right)^{2} L+L^{2}\left(D_{i}+D_{j}\right)|\sin \gamma|,
$$

where $\gamma$ is the angle between the orientational vectors $\underline{\omega}_{1}$ and $\omega_{2}$ of two arbitrary particles of types $i$ and $j$. Note that the extra term in Eq. (15) compared to Eq. (4) is the last term which depends on the relative orientation. If only the leading terms of the excluded volume [second and third terms in Eq. (15) which depend on the length $L]$ are carried forward, the free-energy density of a system of thick and thin hard rods can be obtained from Eqs. (1) and (3) as

$$
\begin{aligned}
a^{*}= & \left(c_{1} \ln c_{1}-c_{1}+c_{2} \ln c_{2}-c_{2}+c_{1} \sigma\left[f_{1}\right]+c_{2} \sigma\left[f_{2}\right]\right) \\
& +\left(c_{1}^{2}\left\{4+k \rho_{11}\left[f_{1}\right]\right\}+2 c_{1} c_{2}\left\{(1+d)^{2}+0.5(1+d)\right.\right. \\
& \left.\left.\times k \rho_{12}\left[f_{1}, f_{2}\right]\right\}+c_{2}^{2}\left\{4 d^{2}+d k \rho_{22}\left[f_{2}\right]\right\}\right),
\end{aligned}
$$

where $k$ is the aspect ratio $\left(k=L / D_{1}\right)$ and the $\rho_{i j}$ functionals are defined as

$$
\rho_{i j}\left[f_{i}, f_{j}\right]=\frac{4}{\pi} \int|\sin \gamma| f_{i}\left(\underline{\omega}_{1}\right) f_{j}\left(\underline{\omega}_{2}\right) d \underline{\omega}_{1} d \underline{\omega}_{2} \quad(i, j=1,2)
$$

The residual free energy corresponds to the terms of order $O\left(c^{2}\right)$ contained in the second bracket of Eq. (16). There are two molecular parameters in the free-energy expression [Eq. (16)]: the reference aspect ratio $k=L / D_{1}$ and the diameter ratio $d$.

It is interesting to note here that the differences between Eq. (5) and Eq. (16), which stem from the orientational degrees freedom, consist only of terms which are proportional to the orientational functionals $\sigma$ and $\rho$. In previous studies [23-32] it was assumed that the finite size (volume) of the particles does not contribute to the stability of a nematic phase with respect to a phase separation into two nematic phases. However, since the finite volume term of the residual free energy in the freely rotating system corresponds to the residual free energy of the parallel system, and nematicnematic separation was observed in that special case, the assumption must be valid only when the orientationindependent part of the residual of free energy $\left(a_{r e s}\right.$ of parallel rods) can be neglected with respect to the orientationaldependent part. For very large aspect ratios $(k \rightarrow \infty)$ it seems reasonable to neglect the finite volume terms, since $k \rho_{i j}[f]$ may take very large values. This is the case along the isotropic-nematic phase transition curve, where $\rho_{i j}[f]$ is always greater than zero. However, in the case of nematicnematic phase separation, it turns out that $\rho_{i j}[f] \rightarrow 0$ in the limit of $k \rightarrow \infty$, and the product $k \rho_{i j}[f]$ is of the same order of magnitude as the terms coming from the finite size of the particle. In these cases, the finite size of the particles should be taken into account and its aspect ratio should not be subsumed into the reduced density, as Onsager originally proposed for monodisperse systems [2]; this omission has been common practice in subsequent studies for polydisperse fluids [23-32]. In the following, we confirm analytically the unexpected aspect ratio dependence of the $\rho_{i j}$ functional using a trial function solution.

Let us assume that the orientational distribution functions of both components can be approximated by the Onsager trial function [2]

$$
f_{i}(\cos \theta)=\frac{\alpha_{i}}{4 \pi} \frac{\cosh \left(\alpha_{i} \cos \theta\right)}{\sinh \left(\alpha_{i}\right)},
$$

where $\alpha_{i}(i=1,2)$ are variational parameters for components 1 and 2 , and $\theta$ is a polar angle between the nematic director and the orientation of a rod. This function, which gives an isotropic distribution for $\alpha_{i}=0$ and nematic phases of increasing order with increasing $\alpha_{i}$, turns out to be very useful because the integrals of the $\sigma$ and $\rho$ orientational functionals 
can be determined analytically [2]. Expressions for $\sigma$ and $\rho$ in terms of $\alpha_{i}$ in the case of highly ordered nematic phases (for large values of $\alpha_{i}$ ) can be obtained by substituting Eq. (18) into Eqs. (13) and (17), giving

$$
\begin{gathered}
\sigma_{i}=\ln \alpha_{i}-1, \\
\rho_{i j}=\frac{4}{\sqrt{2 \pi}} \sqrt{\frac{1}{\alpha_{i}}+\frac{1}{\alpha_{j}}} \quad(i, j=1,2) .
\end{gathered}
$$

Only the leading term of $\alpha_{i}$ has been kept in the asymptotic expansion method for the $\rho$ functional in terms of the variational parameters. The same results can be obtained using a Gaussian trial function, as was first shown by Odijk and Lekkerkerker [45]; we will from now on therefore refer to these expressions for $\sigma$ and $\rho$ as the Gaussian approximation. The values of the variational parameters $\alpha_{i}$ corresponding to the equilibrium nematic phase are obtained by substituting Eq. (19) into Eq. (16) and taking the minimum of the free energy with respect to $\alpha_{1}$ and $\alpha_{2}$; we find that these are given by

$$
\begin{aligned}
& \alpha_{1}=\frac{2}{\pi} k^{2}\left\{\sqrt{2} c_{1}+(1+d) c_{2} \sqrt{\frac{Q}{Q+1}}\right\}^{2}, \\
& \alpha_{2}=\frac{2}{\pi} k^{2}\left\{\sqrt{2} d c_{2}+(1+d) c_{1} \sqrt{\frac{1}{Q+1}}\right\}^{2},
\end{aligned}
$$

where $Q=\alpha_{2} / \alpha_{1}$ is a measure of the relative ordering of component 2 with respect to component 1 . These two equations are not independent, and a simple equation for $Q$ can be obtained such that

$$
\sqrt{Q}=\frac{\sqrt{2} d x_{2}+(1+d) x_{1} \sqrt{\frac{1}{Q+1}}}{\sqrt{2} x_{1}+(1+d) x_{2} \sqrt{\frac{Q}{Q+1}}} .
$$

As can be seen, this equation is only a function of the mole fractions $x_{1}$ and $x_{2}$, and the diameter ratio $d=D_{2} / D_{1}$, i.e., it is independent of the density and aspect ratio $k$. This is very useful because it means that one does not have to recalculate $Q$ at every new density in the phase equilibrium calculation. Since both variational parameters $\left(\alpha_{1}\right.$ and $\left.\alpha_{2}\right)$ must be positive, i.e., $Q>0$, we search only for real positive solutions of Eq. (21) to obtain values of $Q$. It is found that only one solution satisfies this requirement; the values for different compositions and diameter ratios are shown in Fig. 3. It is easy to prove that $Q=1$ for any composition when $d=1$, because the two components are identical in this case. For decreasing diameter ratio, i.e., when the particles of component 1 are thicker than those of component 2, $Q$ decreases very weakly with composition up to $d=0.6$; the dependence of $Q$ on composition becomes more marked for the smaller values of $d$. The most important feature of Fig. 3 is that, in the nematic phase, the thicker rods are always more ordered than the thinner ones at a given composition and density. This is to be expected because the thinner rods occupy less of the total volume than the thicker ones, and as a conse-

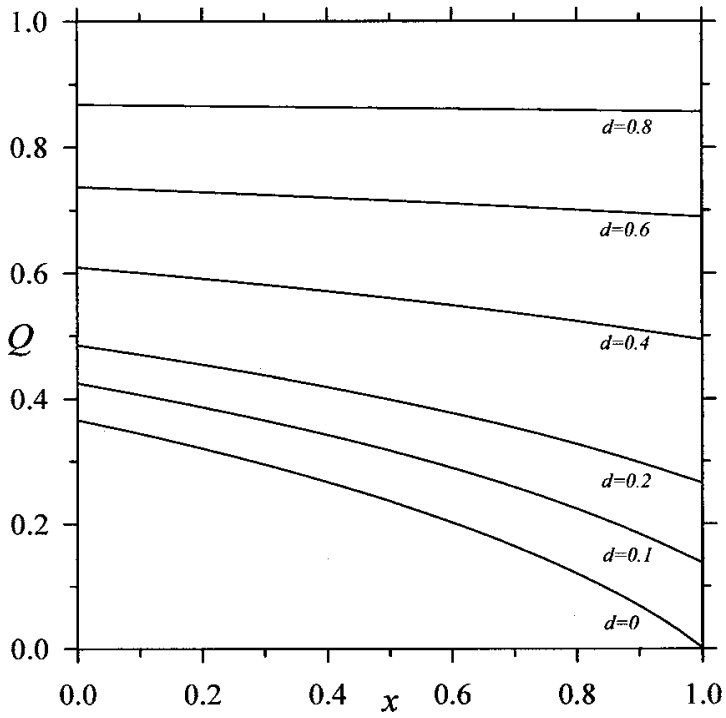

FIG. 3. The relative order parameter $Q$ as a function of mole fraction of thin rods $\left(x=x_{2}\right)$ at some diameter ratio $\left(d=D_{2} / D_{1}\right)$. The resulting curves are the solution of Eq. (21).

quence there is more room for the thinner rods to tilt away from the nematic director than for thicker rods. Results for length-bidisperse hard rods [45] support the above argument, as the longer rods are always more ordered than the shorter ones in the nematic phase. In some special cases the solutions for $Q$ take on a very compact form. For example, in the pure fluid limits $Q=\left(d^{2}+d \sqrt{3 d^{2}+4 d+2}\right) /(1+d)^{2}$ for $x_{2}=1$ $\left(x_{1}=0\right)$, and $Q=\left(-1+\sqrt{2 d^{2}+4 d+3}\right) / 2$ for $x_{2}=0 \quad\left(x_{1}=1\right)$, and in the case of a mixture of thick and infinitely thin rods $(d$ =0) $Q=-x_{1}\left(1-\sqrt{x_{1}^{2}+2 x_{1}}\right) /\left(x_{1}^{2}+2 x_{1}-1\right)$.

More insight on the behavior of the mixture can be gained from the equilibrium thermodynamic properties such as the pressure and chemical potentials. The equilibrium values of the $\sigma$ and $\rho$ functionals can be obtained by substituting the values of $\alpha_{1}$ and $\alpha_{2}$ that minimize the free energy [Eq. (20)] into Eq. (19), resulting in the following equations for $\sigma$ and $\rho$ in terms of densities and molecular parameters:

$$
\begin{gathered}
\sigma_{1}=\ln \left(\frac{2}{\pi}\right)+2 \ln k+2 \ln \left\{\sqrt{2} c_{1}+(1+d) c_{2} \sqrt{\frac{Q}{Q+1}}\right\}-1, \\
\sigma_{2}=\sigma_{1}+\ln Q, \\
k \rho_{11}=\frac{2 \sqrt{2}}{\sqrt{2} c_{1}+(1+d)_{2} \sqrt{\frac{Q}{Q+1}}}, \\
k \rho_{12}=\frac{2 \sqrt{Q+1}}{\sqrt{2} d c_{2}+(1+d) c_{1} \sqrt{\frac{1}{Q+1}}},
\end{gathered}
$$




$$
k \rho_{22}=\frac{2 \sqrt{2}}{\sqrt{2} d c_{2}+(1+d) c_{1} \sqrt{\frac{1}{Q+1}}} .
$$

As expected in this case, we find that each of the equilibrium functionals is a function of the density, diameter ratio, and aspect ratio. Another very interesting result, which can be obtained from Eq. (22) after straightforward but tedious algebra, is that

$$
c_{1}^{2} k \rho_{11}+2 c_{1} c_{2} \frac{1+d}{2} \rho_{12}+c_{2}^{2} d k \rho_{11}=2\left(c_{1}+c_{2}\right)=2 c .
$$

This further suggests that the residual free-energy density of the nematic phase described in terms of the Gaussian approximation does not depend on the assigned aspect ratio, since by substituting Eq. (23) into Eq. (16) we find

$$
a_{r e s}^{*}=2 c+4 c_{1}^{2}+2(1+d)^{2} c_{1} c_{2}+4 d^{2} c_{2}^{2} \text {. }
$$

This equation is different from that derived in a similar way for binary mixtures of hard rods $[25,26,28,45]$ : in these papers the simpler result $a_{\text {res }}^{*}=2 c$ was obtained, as a consequence of neglecting the finite-size terms of the like- and unlike volume contributions of the excluded volumes. By using the simple relation between the pressure and the residual free energy, the reduced pressure can be expressed as

$$
P^{*}=c+a_{r e s}^{*}=3 c+4 c_{1}^{2}+2(1+d)^{2} c_{1} c_{2}+4 d^{2} c_{2}^{2} .
$$

This equation is almost identical to the pressure of the mixture of parallel thick and thin rods [cf. Eq. (7)], with the additional contribution of $2 c$ to the pressure from the orientational degrees of freedom. In agreement with earlier studies, no dependence of the pressure on the aspect ratio is found. It is important to note that in their studies van Roij et al. [31] (who studied this system), Vroege et al. [25] (who considered the length-bidisperse rod systems), and Hemmer [28] (who treated the general mixture of hard rods) give the reduced pressure as $P^{*}=3 c$, which corresponds to the first term in Eq. (25). The difference between the present and the earlier expressions for the pressure has serious implications on the calculation of the phase behavior of the mixture of rods, since the pressure now has an additional composition dependence. When the simpler expression $P^{*}=3 c$ is employed, the densities of the possible coexisting nematic phases $\left(N-N\right.$ demixing) must be identical, since $P_{I}^{*}=P_{I I}^{*}$ in the coexisting phases $I$ and $I I$. The situation is more subtle when the pressure is given by Eq. (25).

Substituting the equilibrium functionals $\sigma$ and $\rho$ given in Eq. (22) into Eq. (16), the reduced equilibrium free-energy density is written as

$$
\begin{aligned}
a^{*}= & {\left[\sum_{i=1}^{2} c_{i} \ln c_{i}+\left(c_{1}+c_{2}\right)\left\{\ln \left(\frac{2}{\pi}\right)+2 \ln k\right\}\right.} \\
& \left.+2\left(c_{1}+c_{2}\right) \ln \left\{\sqrt{2} c_{1}+(1+d) c_{2} \sqrt{\frac{Q}{Q+1}}\right\}+c_{2} \ln Q\right] \\
& +\left[4 c_{1}^{2}+2(1+d)^{2} c_{1} c_{2}+4 d^{2} c_{2}^{2}\right]
\end{aligned}
$$

where the first term in square brackets contains the ideal contribution and the residual contribution from the orientational degrees of freedom, while the second term in square brackets contains the residual free energy of parallel hard rods. The chemical potential is obtained easily form the freeenergy density by differentiating Eq. (16) with respect to component densities

$$
\begin{aligned}
\mu_{1}^{*}= & \left(\frac{\partial a^{*}}{\partial c_{1}}\right)_{c_{2}} \\
= & \ln \left(\frac{2}{\pi}\right)+2 \ln k-1+\ln c_{1} \\
& +2 \ln \left\{\sqrt{2} c_{1}+(1+d) c_{2} \sqrt{\frac{Q}{Q+1}}\right\}+8 c_{1}+2(1+d)^{2} c_{2} \\
& +\frac{4 \sqrt{2} c_{1}+2(1+d) \sqrt{\frac{Q+1}{Q} c_{2}}}{\sqrt{2} c_{1}+(1+d) c_{2} \sqrt{\frac{Q}{Q+1}}}
\end{aligned}
$$

and

$$
\begin{aligned}
\mu_{2}^{*}= & \left(\frac{\partial a^{*}}{\partial c_{2}}\right)_{c_{1}} \\
= & \ln \left(\frac{2}{\pi}\right)+2 \ln k-1+\ln c_{2} \\
& +2 \ln \left\{\sqrt{2} c_{1}+(1+d) c_{2} \sqrt{\frac{Q}{Q+1}}\right\}+\ln Q+8 d^{2} c_{2} \\
& \left.+2(1+d)^{2} c_{1}+\frac{4 \sqrt{2} d c_{2}+2(1+d) \sqrt{Q+1} c_{1}}{\sqrt{Q}\left(\sqrt{2} c_{1}+(1+d) c_{2} \sqrt{\frac{Q}{Q+1}}\right.}\right)
\end{aligned}
$$

Finally, by using the simple relation between the order parameter and the variational parameter obtained by Onsager [2], the order parameters of the nematic phases are given as

$$
\begin{aligned}
& S_{1} \approx 1-\frac{3}{\alpha_{1}}=1-\frac{3 \pi}{2 k^{2}\left\{\sqrt{2} c_{1}+(1+d) c_{2} \sqrt{\frac{Q}{Q+1}}\right\}^{2}} \\
& S_{2} \approx 1-\frac{3}{\alpha_{2}}=1-\frac{3 \pi}{2 k^{2} Q\left\{\sqrt{2} c_{1}+(1+d) c_{2} \sqrt{\frac{Q}{Q+1}}\right\}^{2}}
\end{aligned}
$$

Note that, though the chemical potentials are seen to depend on the aspect ratio $k$, this dependence does not affect the $N$ $-N$ demixing transition because the aspect ratio shifts the chemical potentials by the same constant in both phases. By contrast, the nematic order parameters are seen to be sensitive to the value of the aspect ratio.

In view of the fact that neither the pressure nor the coexistence chemical potentials depends on the aspect ratios of 
the particles, we may conclude that any possible $N-N$ demixing is a function of the diameter ratio only, when the orientational ordering of the system is treated with the Onsager trial function to leading order (Gaussian approximation). It is also important to note that we have assumed the particles to be very long and thin $(k \rightarrow \infty)$, where the first contribution of the excluded volume [Eq. (15)] can be neglected.

On the basis of the spinodal decomposition curve of a fluid of parallel rods, it is reasonable to think that demixing of the nematic phase into two nematic phases may also occur in a system of hard rods with orientational degrees of freedom. Using the pressure [Eq. (25)] and chemical potentials [Eq. (27)] expressions for the nematic phase in the Gaussian approximation, we have explored the possibility of phase separation between two nematic phases for the low values of the diameter ratio where the transition is most likely to take place (see Fig. 2). The resulting binodal curves of the $N-N$ demixing transition are presented in Fig. 4. It can be seen that the additional orientational degrees of freedom of the molecules result in a richer phase diagram than that of the orientationally constrained system (Fig. 2). For example, in the case of the freely rotating system with a diameter ratio of $d=0.2$ the $N-N$ demixing is not found to be associated with a critical point over its entire pressure range; instead a very strong fractionation between a phase which is rich in thick rods and another which is rich in thin rods is observed. The degree of fractionation decreases with increasing diameter ratio over the entire pressure range considered. This tendency is much more pronounced at around $P^{*} \sim 2$, where the phases tend to mix. As a result the single $N-N$ coexistence region observed when $d=0.2$ is seen to divide into two regions for $d \geqslant 0.222$; these two $N-N$ coexistence regions are both delimited by critical points. The existence of the lower critical point is not surprising given the results in Sec. II. In fact, this type of phase behavior has already been observed experimentally in a binary mixture of $f d+f d P E G$ viruses [15]. To the best of our knowledge, no experimental evidence has yet been obtained in colloidal systems for the existence of the region of $N-N$ coexistence ending at an upper critical point (at high pressure and density).

The differences in phase behavior found for the mixtures of parallel and freely rotating rods are a result of the role played by the orientational entropy $(\sim \sigma)$ in stabilizing the mixed state. This is in addition to the subtle competition between entropy of mixing and the packing entropy which was responsible for the demixing in the system of parallel molecules. The freely rotating system can increase its orientational entropy by demixing into thick and thin rich phases, since the thinner rods have more orientational freedom in the phase which is rich in the thin rods than in the phase which is rich in the thick rods (see Fig. 3). As a result, $N-N$ phase separation is predicted at much lower pressures in the freely rotating system than in the parallel system.

In summary, the use of the Onsager theory for the freely rotating systems of thin and thick rods of the same length with the Gaussian approximation appropriate for highly ordered systems leads to large regions of $N-N$ coexistence. Such demixing was also found for the parallel system discussed earlier, but in the case of the freely rotating systems two separate $N-N$ regions (bounded by a "lower" and an
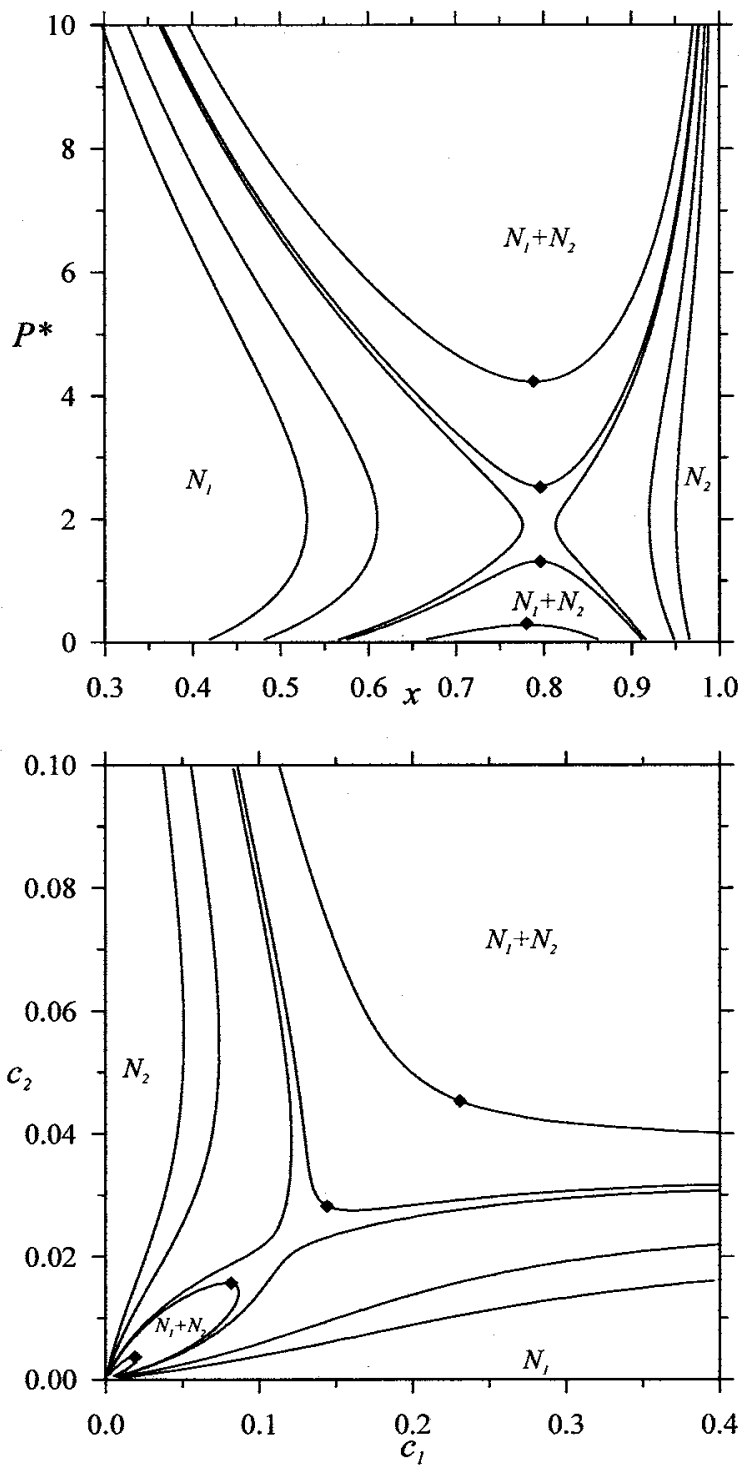

FIG. 4. The pressure-composition $\left(P^{*}-x\right)$ and concentrationconcentration $\left(c_{2}-c_{1}\right)$ representations of the $N-N$ demixing regions of the system of thick (component 1) and thin (component 2) rods according to the Onsager theory at the level of Gaussian approximation [infinite aspect ratio, $k \rightarrow \infty$, Eq. (19)]. The values of the diameter $d=D_{2} / D_{1}$ ratio are the following from outside to inside: $d=0.2,0.21,0.221,0.222$, and 0.23 . The diamond shows the location of the nematic-nematic critical point. The mole fraction is that of the thin rods $\left(x=x_{2}\right)$.

additional "upper" critical point) can be found, and for certain values of the parameters the two regions merge to give a single $N-N$ region with no critical point.

Before moving on to the next section, one should first mention two important failures of the Onsager theory. First, though the Onsager theory is exact in describing the isotropic-nematic phase transition in the limit of infinite aspect ratio (due to the negligible third and higher virial coefficients in the isotropic phase), this is not the case in the dense nematic phase $[46,47]$. In order to assess the implications of this problem with the Onsager theory in relation to our mixture of rods, we now examine the Parsons-Lee exten- 
sion of the theory, which incorporates the contributions of the higher virial terms, albeit in an approximate way. A second and related problem with the Onsager theory is that the packing fraction can be higher than the closed-packed limit for molecules with a finite aspect ratio, which is unphysical. The Parsons-Lee approach circumvents this problem with the use of an accurate hard-sphere reference term.

\section{B. The Parsons-Lee approach}

According to the theory of Parsons [43] and Lee [44] (PL), the residual free-energy density of the system can be written in a very similar to that of Eq. (3), but with

$$
a_{\text {res }}=\frac{a_{r e s}^{H S}}{\rho^{2} B_{2}^{H S}} \sum_{i, j=1}^{2} B_{2}^{i j} \rho_{i} \rho_{j},
$$

where $a_{\text {res }}^{H S}$ is the residual free-energy density and $B_{2}^{H S}$ the second virial coefficient of an effective pure-component hard-sphere fluid. The volume of the effective hard sphere is determined from $v_{H S}=x_{1} v_{1}+x_{2} v_{2}$. The free-energy density of the system in the limit of infinite aspect ratio (Onsager limit, $k \rightarrow \infty$ ) can be expressed as

$$
\begin{aligned}
a^{*}= & c_{1} \ln c_{1}-c_{1}+c_{2} \ln c_{2}-c_{2}+c_{1} \sigma\left[f_{1}\right]+c_{2} \sigma\left[f_{2}\right] \\
& +z\left(c_{1}^{2}\left\{4+k \rho_{11}\left[f_{1}\right]\right\}+2 c_{1} c_{2}\left\{(1+d)^{2}\right.\right. \\
& \left.\left.+0.5(1+d) k \rho_{12}\left[f_{1}, f_{2}\right]\right\}+c_{2}^{2}\left\{4 d^{2}+d k \rho_{22}\left[f_{2}\right]\right\}\right),
\end{aligned}
$$

where $z=(1-0.75 \eta) /(1-\eta)^{2}$ [44] and $\eta=c_{1}+c_{2} d^{2}$ is the packing fraction for a system of thick and thin rods in the Onsager limit. Note that the PL free-energy density [Eq. (30)] differs from the Onsager expression [Eq. (16)] only by the factor of $z$ on the residual contribution, i.e., Eqs. (16) and (30) become identical for very low values of packing fraction $(\eta \rightarrow 0)$. Since the $I-N$ phase transition takes place at vanishingly small packing fraction in the Onsager limit, the two theories give the same results for the $I-N$ transition properties, as desired. However, a nematic-smectic $A$-phase transition can occur at nonzero packing fraction even in the Onsager limit as shown by Poniewierski [48]; and similarly, we do not expect the description of the $N-N$ demixing transition to be equivalent in the two approaches since it occurs at a nonvanishing density.

On searching for the minimum of Eq. (30) with respect to the variational parameters and using the expressions for the orientational functionals given in Eq. (19), we obtain the relation

$$
\begin{aligned}
& \alpha_{1}=\frac{2}{\pi} z^{2} k^{2}\left\{\sqrt{2} c_{1}+(1+d) c_{2} \sqrt{\frac{Q}{Q+1}}\right\}^{2}, \\
& \alpha_{2}=\frac{2}{\pi} z^{2} k^{2}\left\{\sqrt{2} d c_{2}+(1+d) c_{1} \sqrt{\frac{1}{Q+1}}\right\}^{2},
\end{aligned}
$$

for the equilibrium variational parameters of the nematic phase. Interestingly, it can be seen that the ratio of $\alpha_{2}$ to $\alpha_{1}$ is again equal to that given by Eq. (21), which makes the calculations very similar to those using the Onsager theory. As

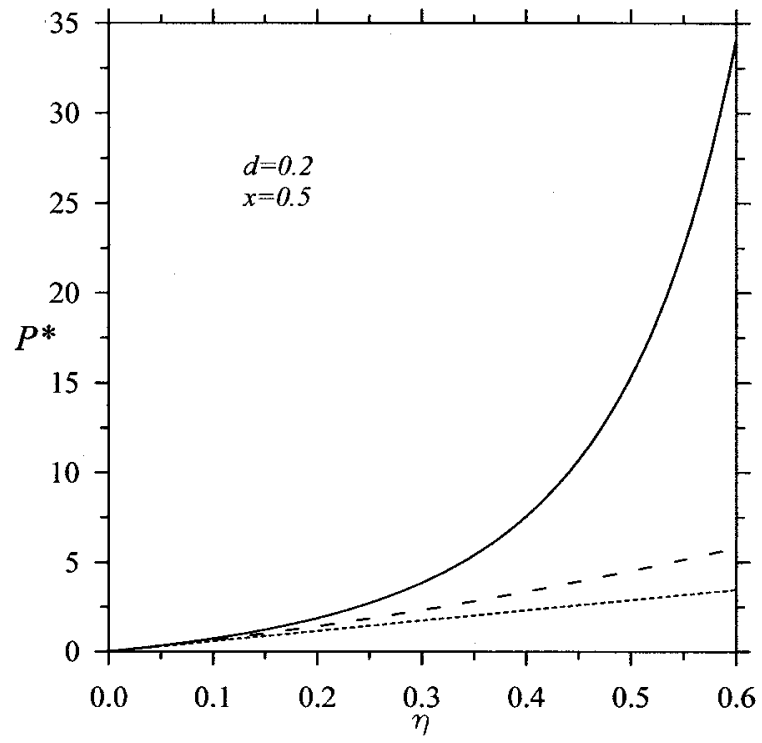

FIG. 5. The pressure-packing fraction $\left(P^{*}-\eta\right)$ representation of the equation of state of a binary mixture of thick (component 1 ) and thin (component 2) rods at equimolar composition for a diameter ratio of $d=D_{2} / D_{1}=0.2$. The continuous curve corresponds to the results of Parsons-Lee theory [Eq. (33)], the dashed curve represents the Onsager theory [Eq. (25)], and the short dashed curve the Onsager theory where finite size effects are not taken into account $\left(P^{*}=3 c\right)$. All curves are obtained using the Gaussian approximation [infinite aspect ratio, $k \rightarrow \infty$, Eq. (19)].

before, the thicker rods are found to always be more ordered than the thinner ones at given composition and density for systems in the Onsager infinite aspect ratio limit. Following the same procedure as in the previous section, it is straightforward to show that the residual free energy can be expressed as

$$
a_{r e s}^{*}=2 c+z\left(4 c_{1}^{2}+2(1+d)^{2} c_{1} c_{2}+4 d^{2} c_{2}^{2}\right),
$$

which is found to differ from that given in Eq. (24) only by the prefactor $z$. The pressure can be also written in a compact form, though it is more complex than the Onsager form due to the dependence of $z$ on the density

$$
\begin{aligned}
P^{*} & =c+a_{r e s}^{*}\left(1+\frac{\eta}{z} \frac{\partial z}{\partial \eta}\right) \\
& =c+\left\{2 c+z\left(4 c_{1}^{2}+2(1+d)^{2} c_{1} c_{2}+4 d^{2} c_{2}^{2}\right)\right\} \frac{4-2 \eta}{4-7 \eta+3 \eta^{2}} .
\end{aligned}
$$

The equilibrium free energy and the chemical potentials can be obtained as described in the previous section

$$
\begin{aligned}
a^{*}= & {\left[\sum_{i=1}^{2} c_{i} \ln c_{i}+\left(c_{1}+c_{2}\right)\left\{\ln \left(\frac{2}{\pi}\right)+2 \ln (k z)\right\}\right.} \\
& \left.+2\left(c_{1}+c_{2}\right) \ln \left\{\sqrt{2} c_{1}+(1+d) c_{2} \sqrt{\frac{Q}{Q+1}}\right\}+c_{2} \ln Q\right] \\
& +z\left[4 c_{1}^{2}+2(1+d)^{2} c_{1} c_{2}+4 d^{2} c_{2}^{2}\right],
\end{aligned}
$$




$$
\begin{aligned}
\mu_{1}^{*}= & \left(\frac{\partial a^{*}}{\partial c_{1}}\right)_{c_{2}} \\
= & \ln \left(\frac{2}{\pi}\right)+2 \ln (k z)-1+\ln c_{1} \\
& +2 \ln \left\{\sqrt{2} c_{1}+(1+d) \sqrt{\frac{Q}{Q+1}} c_{2}\right\}+8 z c_{1} \\
& +2(1+d)^{2} z c_{2}+z \frac{4 \sqrt{2} c_{1}+2(1+d) \sqrt{\frac{Q+1}{Q}} c_{2}}{\sqrt{2} c_{1}+(1+d) \sqrt{\frac{Q}{Q+1}} c_{2}} \\
& +a_{\text {res }}^{*} \frac{5-3 \eta}{(1-\eta)(4-3 \eta)},
\end{aligned}
$$

and

$$
\begin{aligned}
\mu_{2}^{*}= & \left(\frac{\partial a^{*}}{\partial c_{2}}\right)_{c_{1}}=\ln \left(\frac{2}{\pi}\right)+2 \ln (k z)-1+\ln c_{2} \\
& +2 \ln \left\{\sqrt{2} c_{1}+(1+d) \sqrt{\frac{Q}{Q+1}} c_{2}\right\}+\ln Q+8 d^{2} z c_{2} \\
& +2(1+d)^{2} z c_{1}+z \frac{4 \sqrt{2} d c_{2}+2(1+d) \sqrt{Q+1} c_{1}}{\sqrt{Q}\left(\sqrt{2} c_{1}+(1+d) \sqrt{\frac{Q}{Q+1}} c_{2}\right)} \\
& +d^{2} a_{\text {res }}^{*} \frac{5-3 \eta}{(1-\eta)(4-3 \eta)} .
\end{aligned}
$$

It is important to point out, however, that as in the case of Eq. (27), the dependence of the chemical potentials [Eq. (35)] on the aspect ratio only involves the constant logarithmic term, and so has no effect on the phase behavior of the mixture.

Before presenting the results of the demixing analysis that one obtains with the PL free energy [cf. Eq. (30)], we examine the quantitative difference between the pressure of the Onsager theory [Eq. (25)] and that of the PL theory [Eq. (33)]; this is presented in Fig. 5. For the sake of completeness, we also show the pressure of the Onsager theory without finite size effects first derived by Odijk and Lekkerkerker [45]. It can be seen that for low packing fractions (up to $\eta$ $=0.1$ ) the agreement between the various approaches is reasonable; the deviation becomes more and more significant with increasing packing fraction (more than $50 \%$ at $\eta=0.3$ ). It is also clear that, while the PL equation of state [Eq. (33)] diverges when $\eta \rightarrow 1$, the pressure of the Onsager theory [Eq. (25)] remains finite even at close packing; this unphysical behavior of the Onsager theory is a direct consequence of neglecting the virial coefficients above the second.

Nematic-nematic demixing is also predicted by the PL theory as shown in Fig 6. Qualitatively there is no significant difference between these results and those of the Onsager theory. However, the quantitative differences may be quite important from an experimental point of view; the most important feature is that the demixed state is predicted to be
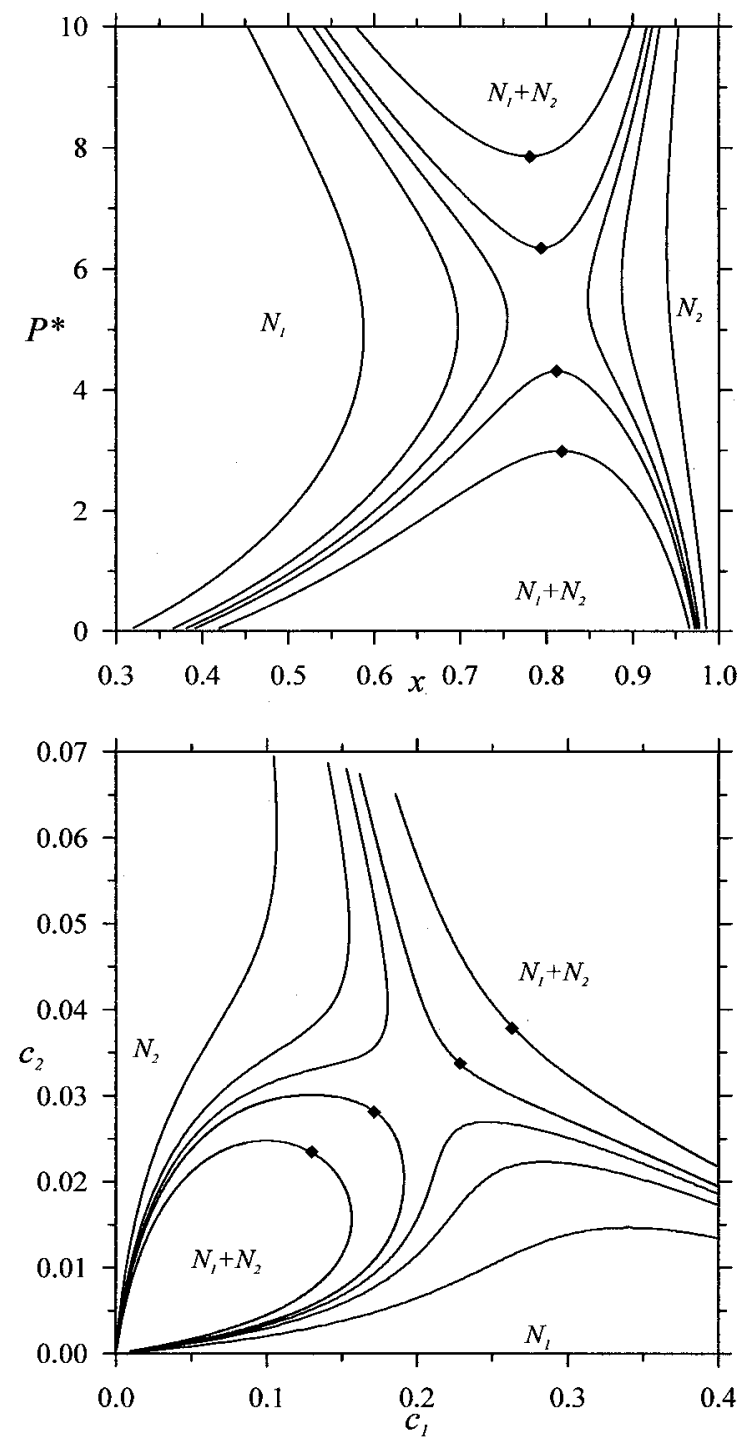

FIG. 6. The pressure-composition $\left(P^{*}-x\right)$ and concentrationconcentration $\left(c_{2}-c_{1}\right)$ representations of the $N-N$ demixing transition of the system of thick (component 1) and thin (component 2) rods according to the Parsons-Lee theory using the Gaussian approximation [infinite aspect ratio, $k \rightarrow \infty$, Eq. (19)]. The values of the diameter ratio $d=D_{2} / D_{1}$ are the following from outside to inside: $d=0.18,0.19,0.193,0.195$, and 0.2 . The diamond shows the location of the nematic-nematic critical point. The mole fraction is that of the thin rods $\left(x=x_{2}\right)$.

less extensive with the PL theory than with the Onsager theory. With the Onsager theory one predicts nematicnematic demixing over the entire range of pressures for $d$ $<0.222$, while in the PL theory this is predicted for $d$ $<0.195$; to stabilize the $N-N$ demixing transition larger differences in shape anisotropies between the thick and thin particles are necessary with the PL theory than the Onsager theory. On the other hand, the range of stability of the $N-N$ coexistence region which is bounded by the lower critical pressure is predicted to extend to much lower pressures and densities in the case ofthe Onsager theory. As a result, the upper critical point which indicates the appearance of the second region of $N-N$ demixing at lower pressures is found 
at much lower pressure and density regimes with the Onsager theory than with the PL theory. As mentioned earlier, this lower region of $N-N$ separation has not yet been observed experimentally [15]. The fact that with the Onsager theory one favors a $N-N$ demixing region bounded by a lower critical point rather than by an upper critical point may lead to the erroneous suggestion that the theory is more appropriate for comparisons with experiment. However, the application of the Onsager theory in the parameter space relevant to the experiment systems is not fruitful as it cannot be extended successfully from the Onsager limit of particle aspect ratio even for the monodisperse systems [46,47]. By contrast, the PL theory gives very accurate results for the properties of the isotropic-nematic phase transition of hard rods from moderate to very high values of the aspect ratio $(5<L / D<\infty)[49,50]$, while the theory of Onsager only becomes accurate from about $L / D>100$ [47]. In view of the results presented in Fig. 6, and considering the other points which have been highlighted, we believe that PL theory provides a more suitable approach to study the phase behavior of mixtures of thick and thin rods both in the isotropic and nematic phases.

\section{The effect of higher order terms (in 1/ $\alpha_{i}$ ) in the Onsager theory}

Trial function methods have been shown to be accurate for the description of the isotropic-nematic phase transition [47]. In the case of nematic-nematic demixing transitions in mixtures of hard rods, recent studies have pointed out that the Gaussian approximation may not be adequate [27,35]. An issue of contention with the use of Onsager and related theories relates to the possibility of obtaining nematic-nematic coexistence regions bounded by a critical point when finite size effects are not incorporated in the description. The fully numerical solution of the free-energy functional [27] suggests that there is a problem with the Onsager trial function, since the numerical calculations confirm the existence of a critical point associated with a $N-N$ phase transition, while calculations using the Onsager trial function do not $[25,28]$. This apparent misconception about the use of the Onsager trial function has been clarified in a recent study by FrancoMelgar et al. [51], who show that very good agreement can be found between the numerical and the trial function methods if higher order terms in $1 / \alpha_{i}$ are incorporated in orientational integrals of Eq. (19). In view of these results, the reliability of our theoretical findings may also be brought into question, and hence a test of the effect of the higher order terms in $1 / \alpha_{i}$ is necessary. Following [2], the expressions of the $\rho_{i j}$ functionals $(i, j=1,2)$, which would be reliable both for $I-N$ and $N-N$ phase transitions, are given as

$$
\begin{aligned}
\rho_{i j}= & \frac{4}{\sqrt{2 \pi}} \sqrt{\frac{1}{\alpha_{i}}+\frac{1}{\alpha_{j}}}\left\{1-\frac{3}{8}\left(\frac{1}{\alpha_{i}}+\frac{1}{\alpha_{j}}+\frac{1}{\alpha_{i}+\alpha_{j}}\right)\right. \\
& \left.+\frac{15}{128}\left(\frac{8}{\alpha_{i} \alpha_{j}}-\left(\frac{1}{\alpha_{i}}+\frac{1}{\alpha_{j}}+\frac{1}{\alpha_{i}+\alpha_{j}}\right)^{2}\right)\right\} .
\end{aligned}
$$

Using this equations instead of Eq. (19b), we obtain rather lengthy equations for the pressure and the chemical poten-
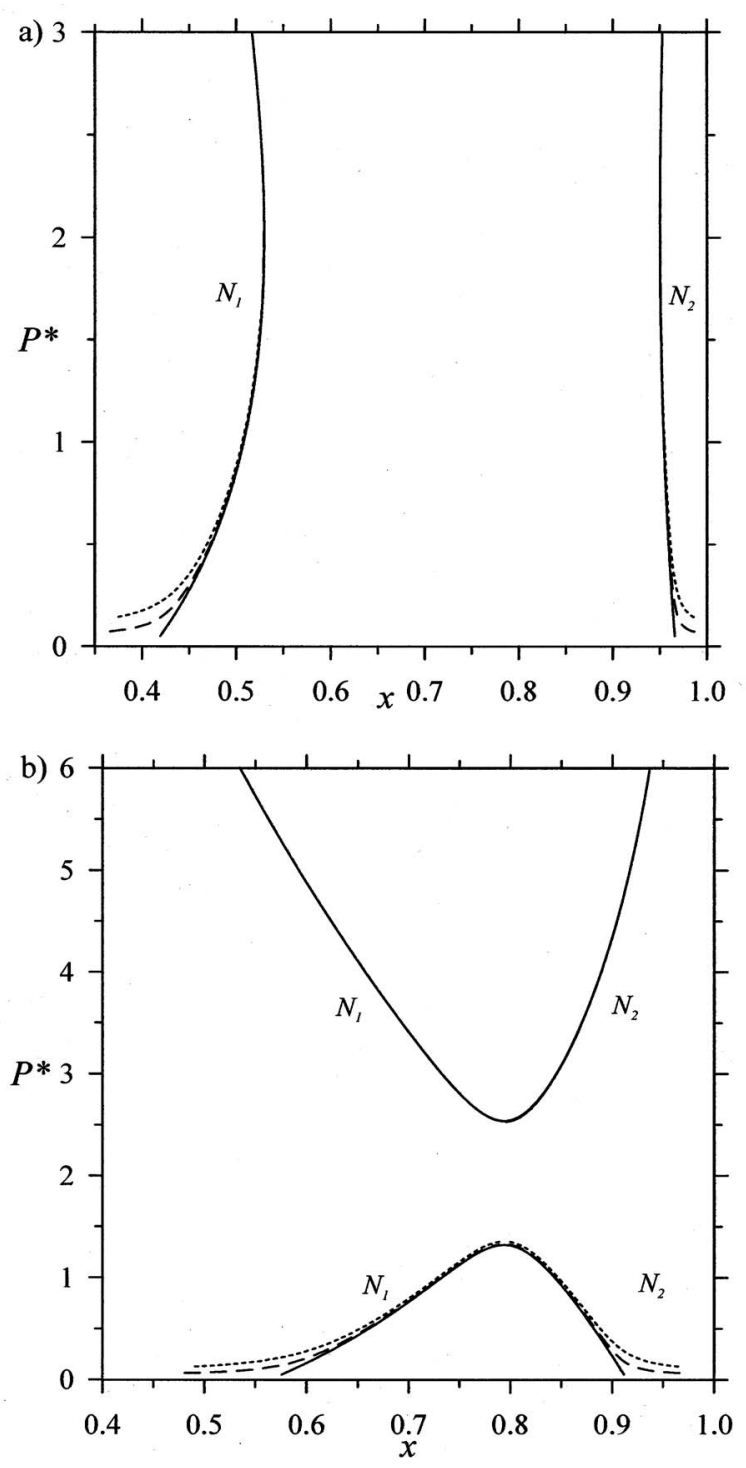

FIG. 7. The effect of higher order terms in $1 / \alpha_{i}$ of the trial function method on the pressure-composition $\left(P^{*}-x\right)$ representation of the nematic-nematic phase boundary of the system of thick (component 1) and thin (component 2) rods for diameter ratios $d$ $=D_{2} / D_{1}$ of (a) $d=0.2$ and (b) $d=0.222$. The continuous curves represent the result of the Gaussian approximation (infinite aspect ratio, $k \rightarrow \infty)$, while the results of inclusion of higher order terms are represented by the dashed curve for aspect ratios of $k=L / D_{1}$ $=1000$ and short dashed curve for $k=500$. The mole fraction is that of the thin rods $\left(x=x_{2}\right)$.

tials, which are not presented here. It is important to mention, however, that both the pressure and chemical potential now exhibit a nontrivial dependence on the aspect ratios which affects the $N-N$ phase separation even for very large aspect ratios. This is an important difference since the use of the Gaussian approximation within the Onsager theory suggests that the pressure and difference in chemical potential do not depend on the aspect ratio as discussed in Sec. III A. In Fig. 7 a comparison of the $N-N$ demixing boundaries obtained with the Gaussian approximation [Eq. (19)] and with the expression [Eq. (36)] is shown for two diameter ratios ( 


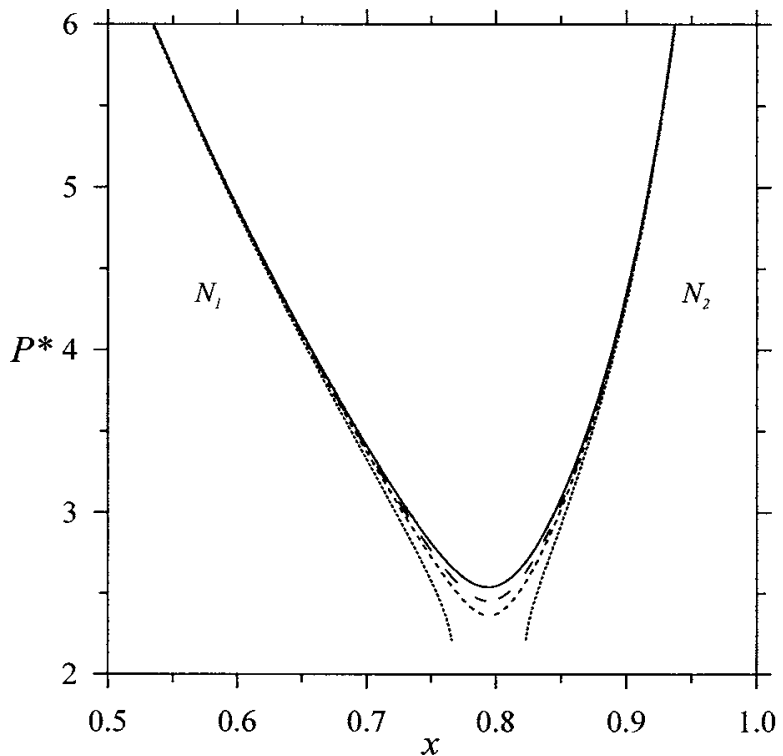

FIG. 8. The effect of the aspect ratio on the pressurecomposition $\left(P^{*}-x\right)$ representation of the nematic-nematic lower critical point of the system of thick (component 1) and thin (component 2) rods using the Onsager trial function with inclusion of higher order terms in $1 / \alpha_{i}$ [Eq. (36)] for diameter ratios $d$ $=D_{2} / D_{1}=0.222: k=1000$ (continuous curve), 200 (long dashed curve), 150 (short dashed curve), and 100 (dotted curve). The mole fraction is that of the thin rods $\left(x=x_{2}\right)$.

$d=0.2$ and 0.222 ) and varying aspect ratios (for the higher order expressions). As expected, no difference can be observed between the two calculations for either of the two diameter ratios or aspect ratios at high pressures where the phases are extremely ordered. This suggests that the equilibrium values of $\alpha_{i}$ parameters are so high that the higher order $1 / \alpha_{i}$ terms of Eq. (36) are negligible, and Eq. (19) provides a good approximation of the orientational order. Differences between the two approximations become apparent, however, at low pressures $\left(P^{*}<1\right)$, where the phases are less ordered and the higher order terms in $1 / \alpha_{i}$ make a nonvanishing contribution to the residual free energy. This effect is more visible with decreasing aspect ratio, and as a result the twophase region obtained using Eq. (36) is seen to broaden.

On first inspection of Fig. 7(b), it is reasonable to question if the $N-N$ demixing region bounded by the lower critical point is independent of the aspect ratio. The answer to this can be gleaned from Fig. 8, where the effect of a decreasing aspect ratio on the high-pressure $N-N$ region can be seen more clearly by considering shorter aspect ratios. The main change is seen close to the critical point, where the pressure is relatively low. By contrast, no significant difference is seen at high pressures even for the system with the shortest aspect ratio considered, $k=100$. It is, nevertheless, evident that the Gaussian approximation predicts a qualitatively incorrect result for the system with $k=100$ : two separate $N-N$ regions with lower- and upper critical points are not predicted with the higher order solution for this system, instead of which a single $\mathrm{N}-\mathrm{N}$ region is predicted over the entire pressure range considered. This means that it is important to incorporate the higher order terms of $1 / \alpha_{i}$ in Eq. (36) to get
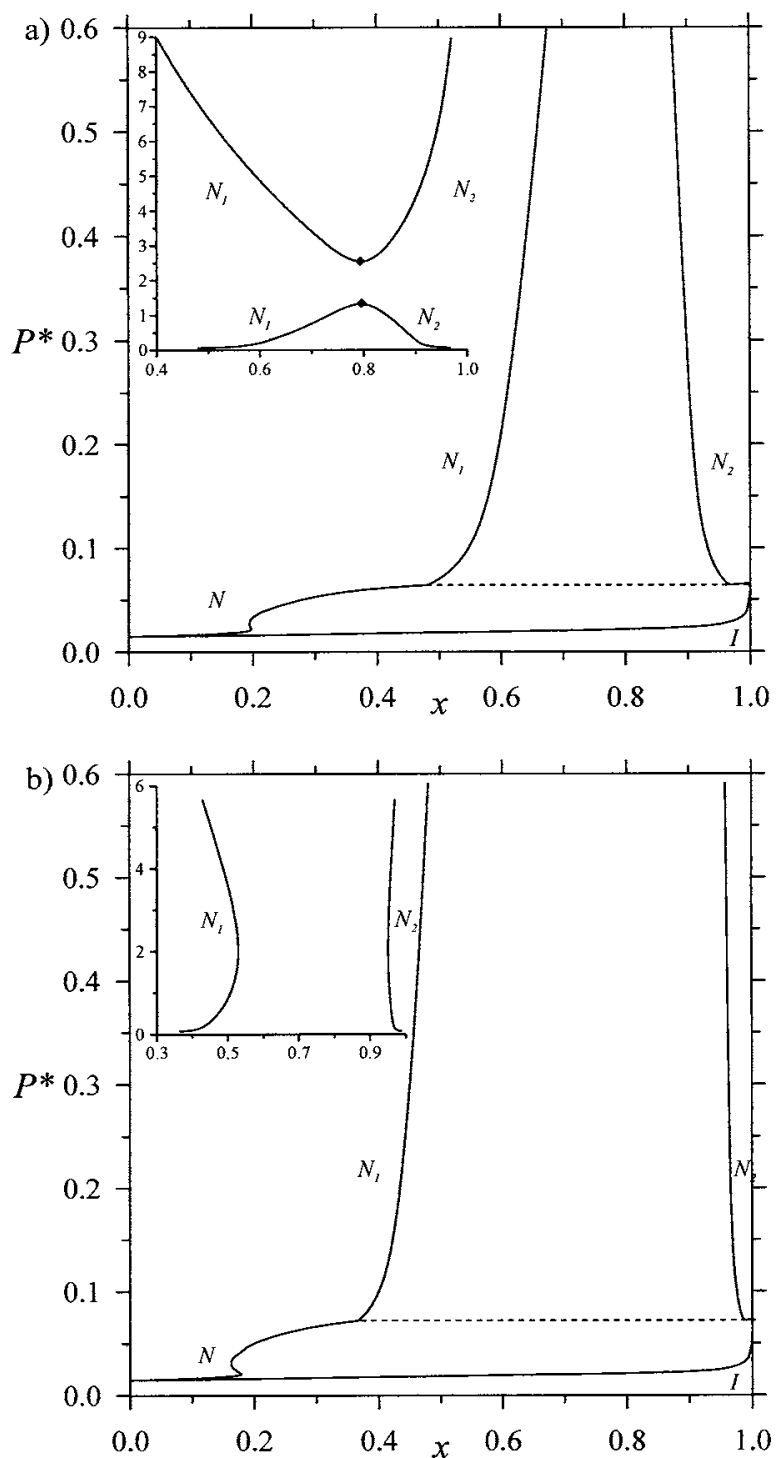

FIG. 9. Reduced pressure-composition $\left(P^{*}-x\right)$ representations of the phase diagram of the system of thick (component 1) and thin (component 2) rods obtained with the Onsager theory at aspect ratio $k=L / D_{1}=1000$ : for diameter ratios (a) $d=D_{2} / D_{1}=0.222$ and (b) $d$ $=0.2$. The resulting phase diagrams are obtained by Onsager trial function method. The continuous curves correspond to the phase boundaries of the isotropic-nematic transition and nematic-nematic demixing transitions, while the dashed line represents the isotropicnematic-nematic three-phase lines. The insets show the nematicnematic phase boundary in the region of higher pressures. The diamonds show the location of the nematic-nematic critical point, while the labels $I$ and $N$ denote isotropic and nematic phases, respectively. The mole fraction is that of the thin rods $\left(x=x_{2}\right)$.

reliable results at low pressures in mixtures comprising molecules of shorter aspect ratios, where the rods are not as highly ordered. This is also the case for the $N-N$ coexistence region bounded by a higher critical point [51].

Using the more accurate representation of the $\rho_{i j}$ functionals [Eq. (36)], we have determined the full phase diagram of the system at $k=1000$ (see Fig. 9), a large aspect ratio where the Onsager second virial theory is practically exact for the isotropic and moderately ordered nematic phases. It can be 
seen that the isotropic phase is only stable at very low values of the pressure and density. Along the $I-N$ phase coexistence curve, the pressure and the corresponding densities are still very low, implying that the Onsager theory preserves its accuracy. Though it is customary to rescale the density and the pressure by multiplying them with the aspect ratio to make the region of the $I-N$ transition more visible [2], for our purposes this type of rescaling is not useful because the coexisting nematic phases are far from being dilute (see Figs. 4 and 6). As found in earlier studies [23-28], a strong fractionation and a reentrant nematic phase characterizes the $I-N$ phase separation. For a diameter ratio of $d=0.2$ the finite size effect hardly affects the phase behavior of the system, except that the bottleneck shape of the $N-N$ phase separation [see the inset of Fig. 9(b)] cannot be reproduced with the traditional Onsager approach [25], where only the leading term in the excluded volume is incorporated. The finite size effects are found to be more significant for the mixture with a diameter ratio of $d=0.222$, where one predicts that the $N-N$ phase coexistence curve starting from the $I-N-N$ three phase line is closed off by a critical point at higher pressure. Above this upper critical point the system mixes in all proportions over a narrow pressure range of $1.33<P^{*}<2.53$, and then demixes again above the lower critical pressure of $P^{*}=2.53$. The qualitatively different result of a single $N-N$ coexistence region with no critical points was found by van Roij et al. [26] when only the leading term of the excluded volume is retained (no finite size contributions).

Summing up to this point, we have so far been able to show that the orientational entropy arising from molecular rotational freedom does not prevent the existence of $N-N$ demixing which was predicted for the system of parallel rods. In fact, the extent of demixing is enhanced, shifting the lower critical point towards lower pressures, and giving rise to a new $N-N$ demixing region emerging from low pressure. We have attributed this type of phase behavior to the fact that the orientational entropy can increase substantially through demixing into two phases, one rich in thick rods and the other rich in thin rods. Using the Gaussian trial function approximation, a new analytical equation of state is derived for the nematic phase, which takes into account the finite size effects. Moreover, we show that the Onsager and PL theories are not identical in the nematic phase even in the Onsager limit of infinite aspect ration due the incorporation of the higher virial coefficients in the PL expression (albeit in an approximate manner). The resulting phase diagrams differ only in quantitative, and not qualitative manner. We also shed some light on the importance of including higher order terms in the expansion of the $\rho_{i j}$ functionals and the shortcomings of the Onsager and Parsons-Lee theories. In the following section we carry forward some of these ideas, focusing on approaches which provide the most quantitative description for comparison with experimental systems.

\section{NUMERICAL RESULTS FROM THE PARSONS-LEE THEORY FOR A MIXTURE OF FREELY ROTATING THICK AND THIN HARD RODS}

Though the second virial theory of Onsager can account for the $I-N$ phase coexistence of fluids of mono- and poly- disperse hard rods, it is not useful for practical purposes, as it is only appropriate to describe particles with large aspect ratios $(L / D \geqslant 100)$, i.e., for very long and thin molecules. Moreover, the requirement of extreme particle shapes complicates the simulation of systems in the Onsager limit. Using a special scaling method, Bolhuis and Frenkel [52] have managed to approach the Onsager limit by simulation, and have shown that a system of long rods follows the predictions of Onsager's theory in the isotropic and moderately ordered nematic phases. Simulation studies have also shown that the PL theory can provide very accurate results for the equation of state both in the isotropic and in the nematic phases, and for the $I-N$ transition properties from moderate to large aspect ratios $[49,50]$. These findings, and the fact that the PL theory establishes a bridge between the Onsager theory and the very accurate Carnahan and Starling [53] expression for hard spheres, mean that the PL approach is particularly useful for the description of the $I-N$ phase behavior of hard rods of any aspect ratio.

In this section we calculate the phase diagrams of binary mixtures of thin and thick rods using the PL theory. The model molecular parameters are chosen to represent an experimental system that we have studied recently [15]. The system in question consists of a mixture of thin (charged semiflexible $f d$-virus) and thick ( $f d$-PEG virus, polyethylene glycol-coated $f d$-virus) rods. The aspect ratio of the $f d$-PEG virus is of the order of $k \sim 24$, which suggests that third and higher-body virial coefficients are not negligible [46,47]. The longer (thinner) component ( $f d$-virus) resembles a long rod at very high ionic strengths where its maximum aspect ratio is of order $k \sim 100$, and here the Onsager theory is expected to provide a good description of the $I-N$ transition. In the case of the mixture, however, $N-N$ demixing is also expected and Onsager's theory will not be accurate enough for comparison with the experimental system.

The phase behavior for molecular parameters in the vicinity of the experimental ones is also studied to identify the model system that best reproduces the phase diagram of the $f d-f d$-PEG mixture (see Fig. 1). Before presenting the results, it is important to mention a number of key points. The approximate trial function approach is not employed here; instead, a discrete representation is used to describe the orientational distribution functions of the mixture. A formal minimization of the free-energy functional with respect to the orientational distribution functions is carried out, and the resulting coupled integral equations for $f_{1}(\theta)$ and $f_{2}(\theta)$ are then solved by iteration using Simpson's rule [41]. This procedure is free from approximations, and an accurate implementation of the method should provide essentially exact results within the PL approximation. Details of the method are given in [41]. In accordance with our previous numerical studies $[33,40,41]$, the particles are modeled as hard cylinders instead of hard spherocylinders. The difference between the two models is not significant; the calculated coexisting densities of the $I-N$ transition are almost identical in the two models at any aspect ratio. All the terms in the excluded volume interactions are incorporated in the calculations.

Our goal is to determine all types of phase diagrams (the global phase diagram) possible for the experimental system of thick and thin rods. Since the aspect ratio of the $f d$-PEG 

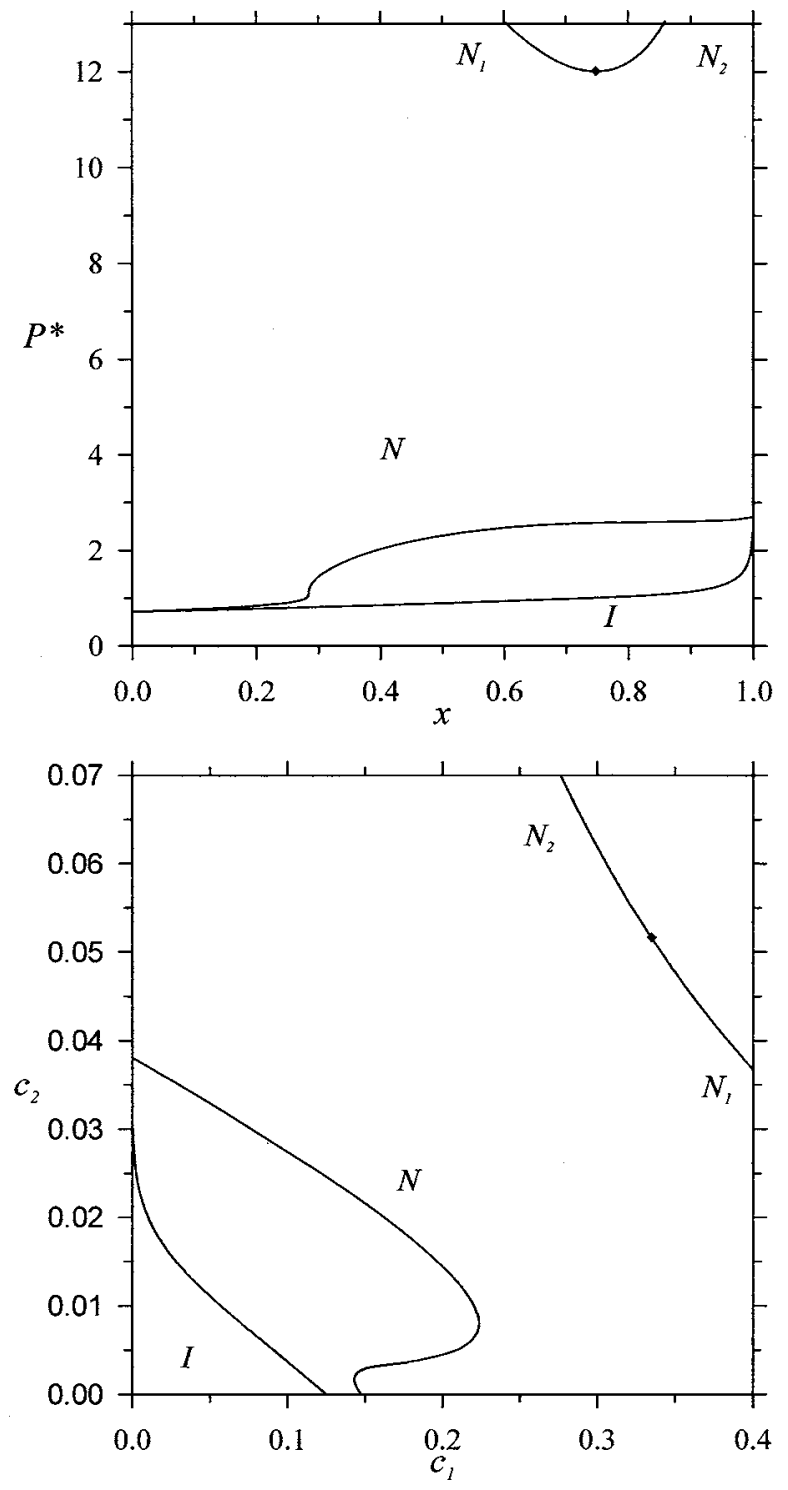

FIG. 10. Type I phase behavior. Reduced pressure-composition $\left(P^{*}-x\right)$ and concentration-concentration $\left(c_{2}-c_{1}\right)$ representations of the phase diagram of the system of thick (component 1$)$ and thin (component 2) rods obtained with the Parsons-Lee theory at aspect ratio $k=L / D_{1}=24$ and diameter ratio $d=D_{2} / D_{1}=0.227$. The continuous curves correspond to the phase boundaries of the isotropicnematic and nematic-nematic phase transitions. The diamond shows the location of the nematic-nematic critical point. The labels $I$ and $N$ denote isotropic and nematic phases, respectively. The mole fraction is that of the thin rods $\left(x=x_{2}\right)$.

rods (component 1 in our study) is always 24 [15], it is reasonable to start by exploring the effect of varying the diameter of the bare $f d$ component on the phase diagram for this aspect ratio. On fixing $k=24$, four distinct types of phase diagrams are found for varying diameter ratios. The first type of phase diagram, which we characterize as type I, is observed for a diameter ratio $d=0.227$. The phase diagram is presented in a pressure-composition and a density-density representation in Fig. 10. In agreement with the experimental results, a region of nematic demixing is obtained bounded by a lower critical point at high densities (pressures). At lower pressures a separate region of isotropic-nematic phase coexistence is seen. In contrast with the experimental data, however, a strong fractionation of the nematic and isotropic phases is predicted [cf. Fig. 1(a)]. The actual experimental diameter ratio is closer to $d=0.345$, so that we could expect better agreement for this value of the diameter ratio. Unfortunately in this case, the stability of the calculated $N-N$ phase transition would be questionable due to the extremely high packing fractions of the nematic phases, which would be more typical of smectic or solid phases. In fact, it should be noted that, even for $d=0.227$, the packing fraction of the lower critical pressure is rather high $\left(\eta_{c}=0.385\right)$.

A different type of phase diagram (which we characterize as type II) is observed at $d=0.2$, where a new $N-N$ phase coexistence region bounded by an upper critical pressure has emerged from the $I-N$ phase boundary (see Fig. 11). The existence of this upper critical point has been the subject of some debate in the past $[23,25,26]$, but the more recent numerical $[27,35]$ and accurate trial function methods [51] have confirmed its existence. Though this type of behavior was not found in the $f d-f d$-PEG experimental system [15], it could be possible in a narrow region of parameter space. For this type of phase behavior, decreasing the diameter ratio leads to a more extensive $N-N$ phase coexistence; the twophase regions cover a larger range of compositions, and the lower and upper critical points are seen to approach each other, and eventually merge. As a result a third type of phase diagram (type III) is obtained for $d=0.192$ (see Fig. 12). This figure resembles that of the experimental system with $d$ $=0.333$, but Figs. 12 and 1(b) are qualitatively different. In addition to the wide two-phase region and reentrance phenomenon of the nematic phase, the theoretical diagram presents an intermediate region where the coexisting nematic phases exhibit a weak tendency to remix, which is not seen in the experimental system.

Isotropic-isotropic $(I-I)$ demixing is obtained when the diameter ratio is further decreased, indicating that the components become even less compatible. The appearance of the (I-I) demixing transition characterizes the fourth type of phase behavior (type IV); it first becomes stable at a diameter ratio of $d \cong 0.148$ for an aspect ratio of $k=24$. To illustrate type IV phase behavior, we examine a system with the following aspect and diameter ratios: $k=5$ and $d=0.2$. The reason for this choice is that these molecular parameters allow details of the demixing transitions to be seen on the same density and pressure scales. The resulting phase diagram is shown in Fig. 13. In addition to the $I-I$ demixing transition, the only new feature of the phase diagram is the continuous widening of the $N-N$ demixing transition at higher pressure (density). Note that the $N-N$ demixing region of the experimental system also exhibits a continuous increase in the extent of $N-N$ phase separation at higher density.

Given that the quantitative differences between theory and experiment are significant, we now undertake a comprehensive study to find molecular parameters for which the theory is capable of at least a qualitative description of the experimental system. In order to do this we determine the ranges of stability of the four different types of phase diagram in the diameter ratio-aspect ratio plane. The boundary between type I and type II phase systems is found by monitoring the 


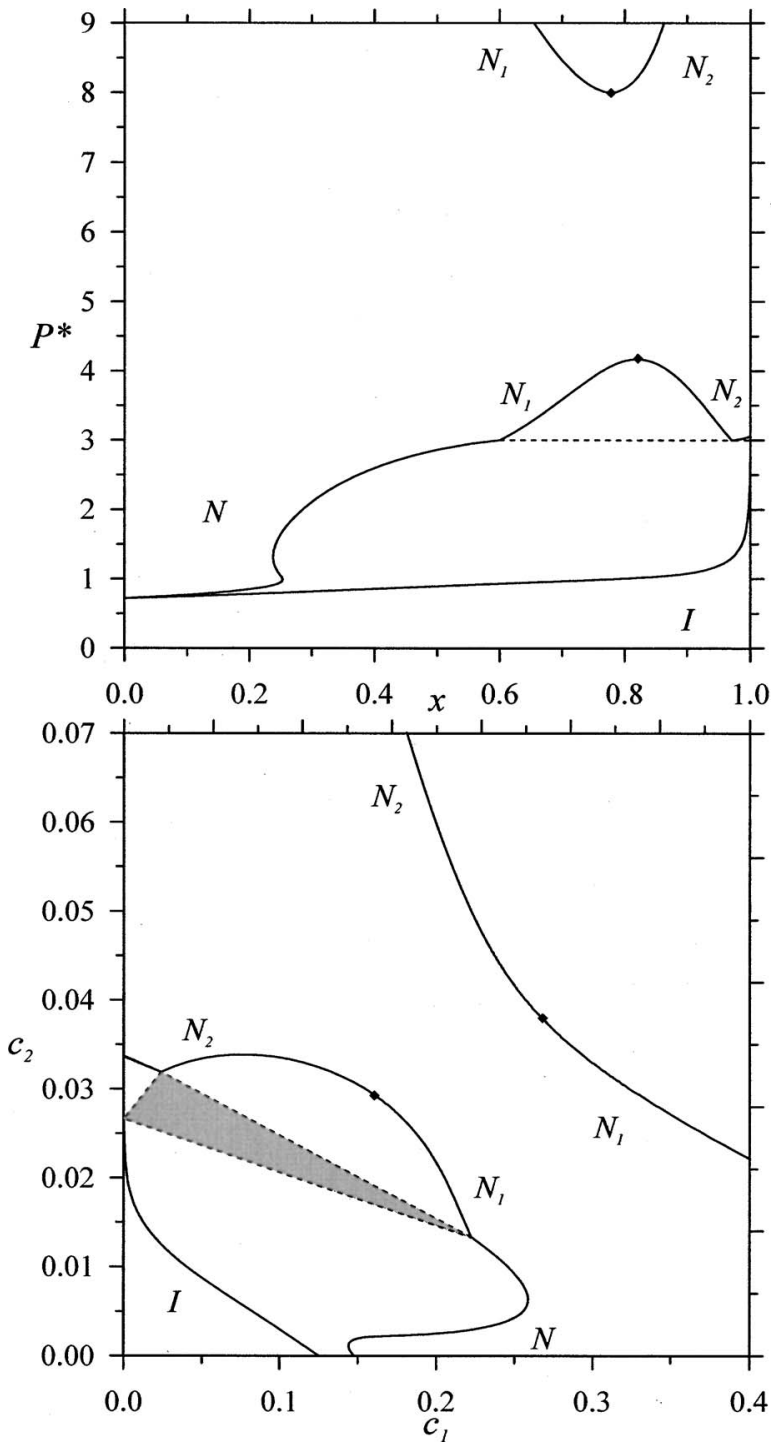

FIG. 11. Type II phase behavior. Reduced pressure-composition $\left(P^{*}-x\right)$ and composition-composition $\left(c_{2}-c_{1}\right)$ representations of the phase diagram of the system of thick (component 1) and thin (component 2) rods obtained with the Parsons-Lee theory at aspect ratio $k=L / D_{1}=24$ and diameter ratio $d=D_{2} / D_{1}=0.2$. The continuous curves correspond to the phase boundaries of the isotropic-nematic and nematic-nematic phase transitions, while the dashed line represents the isotropic-nematic-nematic three-phase lines. The three phase region is indicated by the gray triangle in lower panel (b). The diamonds show the location of the nematic-nematic critical points. The labels $I$ and $N$ denote isotropic and nematic phases, respectively. The mole fraction is that of the thin rods $\left(x=x_{2}\right)$.

position of the $I-N-N$ three-phase line as a function of the diameter ratio at a given aspect ratio. For example, starting from $k=24$ and $d=0.2$, this means that we follow the compositions of the coexisting $N-N$ phases of the $I-N-N$ threephase line with increasing diameter ratio. The diameter ratio where the compositions of the coexisting nematic phases become identical corresponds to the limit of the stability of systems with type II behavior with respect to type I. In a similar manner, we can determine the boundary between systems exhibiting type IV and type III phase behavior by fol-

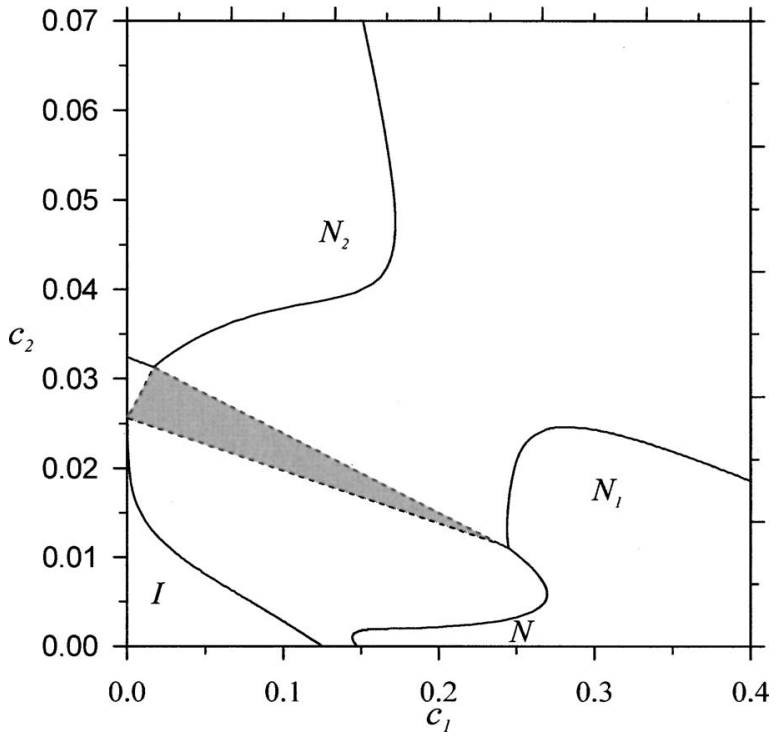

FIG. 12. Type III phase behavior. Composition-composition $\left(c_{2}-c_{1}\right)$ representations of the phase diagram of the system of thick (component 1) and thin (component 2) rods obtained with the Parsons-Lee theory at aspect ratio $k=L / D_{1}=24$ and diameter ratio $d=D_{2} / D_{1}=0.192$. The continuous curves correspond to the phase boundaries of the isotropic-nematic and nematic-nematic phase transitions, while the three-phase region is indicated by the gray triangle. The labels $I$ and $N$ denote isotropic and nematic phases, respectively.

lowing the I-I-N three-phase line. In this case the disappearance of the three-phase line indicates the destabilization of I-I coexistence. The boundary between type II and type III is the most difficult to locate, as it requires the simultaneous determination of the lower and upper critical points. This involves solving the set of criticality conditions [Eqs. (8) and (10)] with the PL free-energy density. While the critical point can be obtained analytically in the isotropic phase, numerical differentiation has to be used to determine the derivatives of the free-energy density for the $N-N$ critical point due to the complex density dependence of the orientational distribution functions in the nematic phase. Starting from a system with type II phase behavior, a gradual decrease of the diameter ratio in steps of $\Delta d=0.005$ allows an accurate determination of the double critical end point where the lower and upper $\mathrm{N}-\mathrm{N}$ critical points merge, which also corresponds to the boundary between systems with type II and III phase behavior. The results of the calculations in the range $4<k<50$ are shown in Fig. 14. The stability of type I phase behaviour is seen to be very sensitive to the aspect ratio; in systems with aspect ratios which are too short or too long the behavior is postponed to large diameter ratios, but it is seen for a wide range of diameter ratios in the case of intermediate particle anisotropies $(k \sim 13)$. Systems which exhibit type II phase behavior are only observed for aspects ratios of $k>7.5$, and thereafter it is seen for systems with a wider range of diameter ratios for increasing aspect ratio. For aspect ratios $k$ $<7.5$ a direct transition from systems with type I to type III behavior is predicted; this is a key finding as this corresponds to the behavior observed experimentally. The comparison between experiment and theory for the transitions 

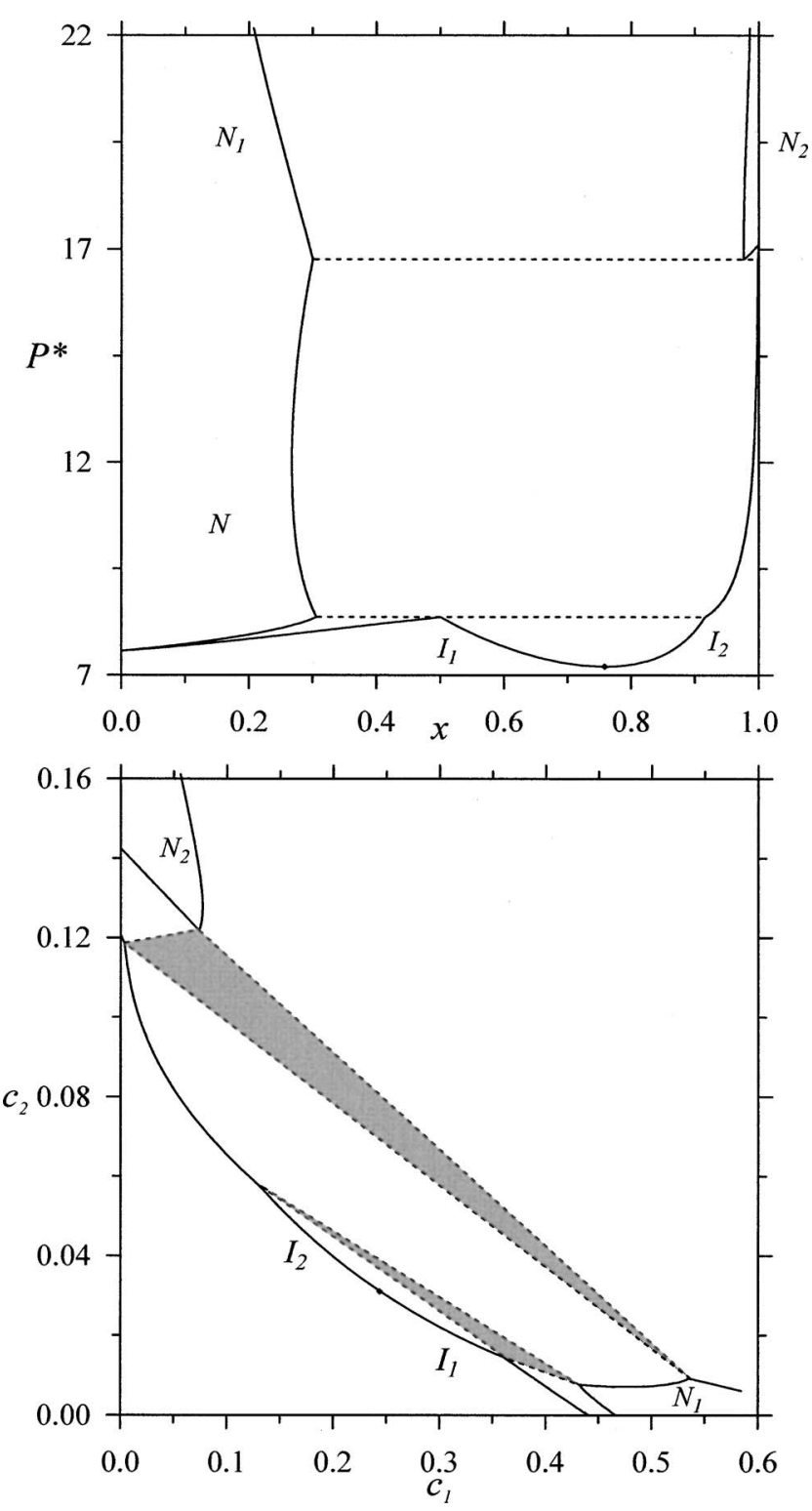

FIG. 13. Type IV phase behavior. Reduced pressurecomposition $\left(P^{*}-x\right)$ and composition-composition $\left(c_{2}-c_{1}\right)$ representations of the phase diagram of the system of thick (component 1) and thin (component 2) rods obtained with the Parsons-Lee theory at aspect ratio $k=L / D_{1}=5$ and diameter ratio $d=D_{2} / D_{1}=0.2$. The continuous curves correspond to the phase boundaries of the isotropic-nematic, isotropic-isotropic, and nematic-nematic phase transitions, while the dashed line represents the isotropic-isotropicnematic and isotropic-nematic-nematic three-phase lines. The threephase region is indicated by the gray triangle in lower panel (b). The diamond indicates the location of the isotropic-isotropic critical point. The labels $I$ and $N$ denote isotropic and nematic phases, respectively. The mole fraction is that of the thin rods $\left(x=x_{2}\right)$.

between phase diagrams with decreasing diameter ratio is most quantitative for an aspect ratio of $k=4$; the calculated phase diagrams are presented in Fig. 15. Comparing Figs. 1 and 15 , it can be seen that the agreement between the theory and the experiment is now much improved: the theoretical $I-N$ phase separation does not present reentrance or strong fractionation. Moreover, the $N-N$ demixing transition is seen

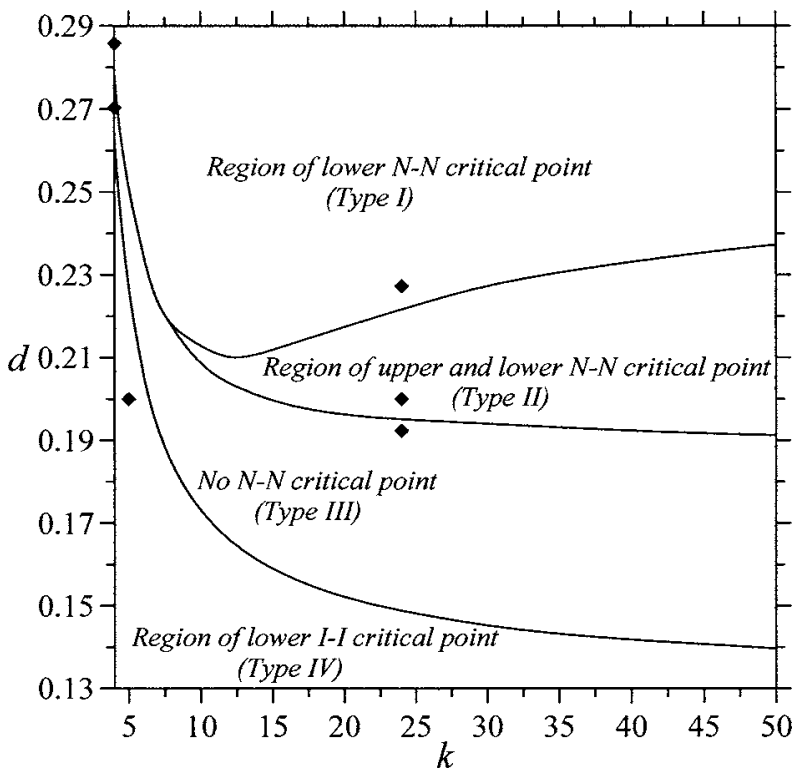

FIG. 14. Classification of the types of phase behavior of the binary mixture of rigid thick (component 1 ) and thin (component 2) rods according to Parsons-Lee theory. Regions of four distinct types of phase diagrams are shown in diameter ratio $\left(d=D_{2} / D_{1}\right)$-aspect ratio $\left(k=L / D_{1}\right)$ plane. The diamonds indicate the molecular parameters of those systems for which the phase diagrams are presented in the subsequent figures.

to widen continuously at higher density. We believe that the reason for the lack of type II phase behavior in the experimental system stems from the increasing impact of the endeffects of the excluded volume interactions with decreasing aspect ratio; it is interesting to note that, while these contributions stabilize the $I-I$ demixing transition, they destabilize the $N-N$ demixing transition. A drawback in our comparison with experiment is that, unfortunately, the molecular parameters used in the theoretical calculations are significantly different from those of the experimental system.

\section{CONCLUSION}

We have studied the phase behavior of binary mixtures of thick and thin hard rods of the same length using the Onsager and Parsons-Lee theories. Two methods have been used to determine the equilibrium free energy and phase behavior of the mixtures for varying molecular parameters: the trial function approach and direct functional minimization. The use of trial functions provides analytical, but approximate, equations for the thermodynamic properties such as the free energy, pressure, and the chemical potentials [Eqs. (25)-(27) in the Onsager theory; Eqs. (33)-(35) in PL theory]; the functional minimization method provides exact solutions for an accurate implementation, but no analytical expressions for the thermodynamics.

By comparing the phase behavior of the parallel and the freely rotating models, we have shown that the excluded volume terms related to configurations of parallel particles also play a crucial role in stabilizing the nematic phase of the freely rotating system. For example, using Onsager's theory 

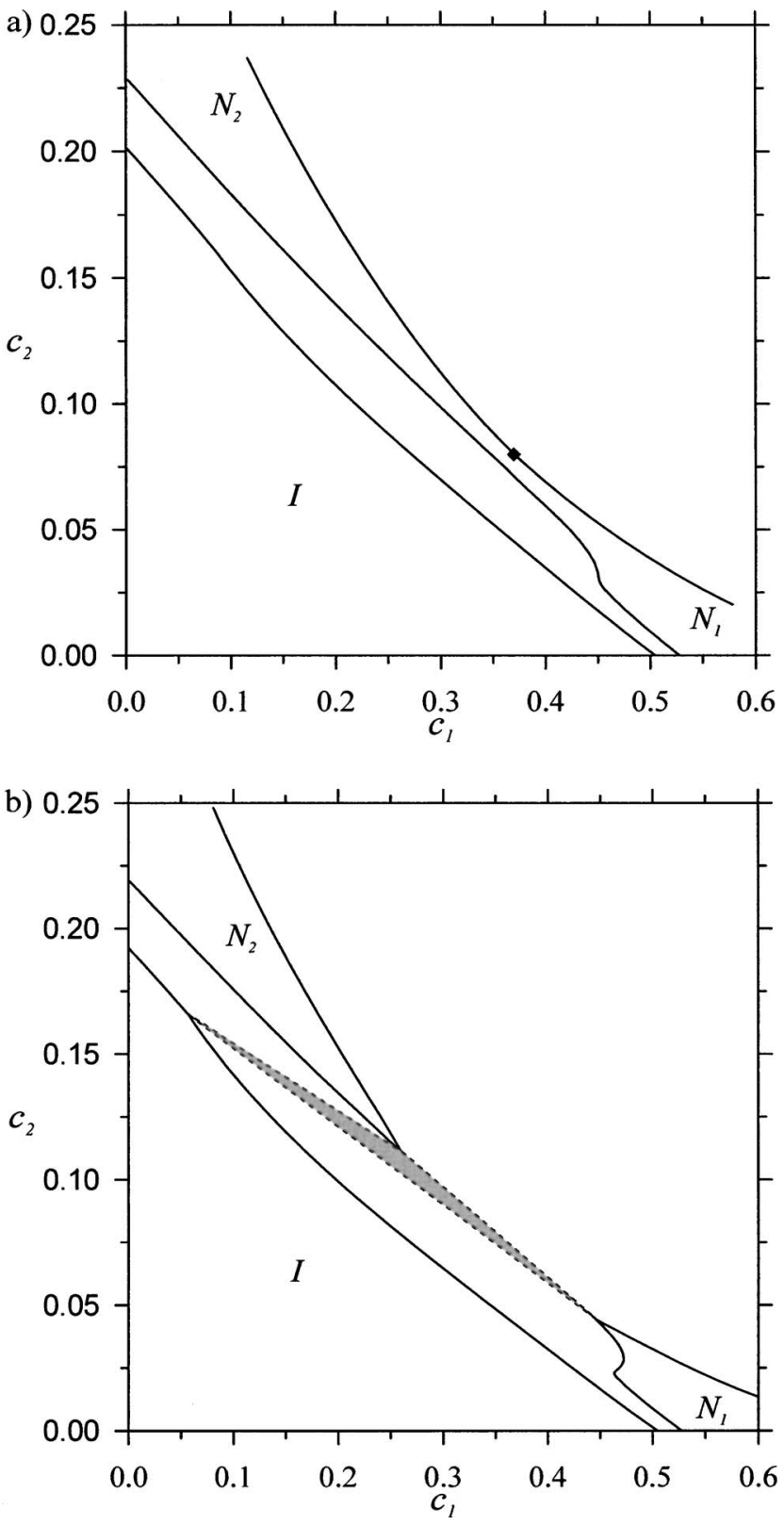

FIG. 15. Composition-composition $\left(c_{2}-c_{1}\right)$ representations of the phase diagram of the system of thick (component 1) and thin (component 2) rods obtained with the Parsons-Lee theory at aspect ratio $k=L / D_{1}=4$ and diameter ratios of $d=0.286$ (a) and $d=0.270$ (b). The continuous curves correspond to the phase boundaries of the isotropic-nematic and nematic-nematic phase transitions. The three-phase region is indicated by the gray triangle in figure (b). The diamonds show the location of the nematic-nematic critical point, while the labels $I$ and $N$ denote isotropic and nematic phases, respectively.

with the Gaussian approximation (large aspect ratios) the pressure [Eq. (25)] is obtained as a sum of two terms: the first is three times the ideal pressure of the freely rotating model, and the second is the contribution due to the particles being parallel. The inclusion of the finite size terms has a dramatic effect on the phase behavior in relation to the stability of the nematic phase. In the case of the system of parallel system of thin and thick rods only a single region of
$N-N$ coexistence is predicted, bounded at lower pressure (density) by a critical point. Depending on the value of the diameter ratio, the freely rotating systems can simultaneously exhibit demixing and enhanced mixing; this can give rise to $N-N$ demixed regions bounded by lower or upper critical points. The $N-N$ demixing transition bounded by an upper critical point is found between two types of phase behavior; it is preempted by an $I-N$ phase transition when the two particles are not sufficiently dissimilar, while for increasing incompatibility between the components (decreasing diameter) an eggcuplike demixing phase boundary with no critical points is observed. By performing a global search monitoring critical points and three-phase lines, four different types of phase diagrams are predicted: only a lower $N-N$ critical point (type I); lower and upper $N-N$ critical points (type II); $N-N$ demixing without $N-N$ critical points (type III); and only a lower $I-I$ critical point (type IV). Types I and II phase behavior have not been previously seen in theoretical studies of the system of thick and thin rods [22,31].

We have also provided a semiquantitative description of the experimental phase behavior of a mixture of bare and PEG-coated $f d$ viruses. For the precise values of the molecular parameters of the experimental system $(k=24, d=0.345$; and $k=24, d=0.333)$ the phase diagrams obtained with the PL theory deviate substantially from those determined experimentally. In order to get the closest theoretical representation of the type of behavior found experimentally, we find that the aspect ratio of the particles has to be less than 7.5. This is significantly different from the experimental value. There are at least two possible reasons for this discrepancy. On the one hand the $f d$ and $f d$-PEG viruses are far from being hard rods; they are actually flexible and chiral particles. The $f d$ virus is also negatively charged and the interaction between the $f d$ virus and the $f d$-PEG virus is nonadditive. Furthermore, neither the PL nor the Onsager theory is exact in the nematic phase, even in the Onsager limit. To resolve these problems it would be desirable to perform a thorough simulation study in the dense nematic phase for a mixture of hard rods to test the reliability of the theories. In addition, improvements to the model, i.e., the treatment of flexibility, electrostatic- and nonadditive unlike interactions, should provide a closer representation of the experimental system, though this would involve much more complex theoretical calculations. We leave these issues for future studies.

\section{ACKNOWLEDGMENTS}

S.V. would like to thank the OTKA of Hungary (Grant Number F 47312) for financial support; A.G. would like to thank the Engineering and Physical Sciences Research Council (EPSRC) of the U.K. for support. K.P. and S.F. were supported by NSF/DMR grant. A.G. and G.J. acknowledge further support from the EPSRC (GR/N03358, GR/N35991, and GR/R09497), the Joint Research Equipment Initiative (JREI) for computer hardware (GR/M94427), and the Royal Society-Wolfson Foundation for the award of a refurbishment grant. 
[1] S. Chandrasekhar, Liquid Crystals, 2nd Ed. (Cambridge University Press, Cambridge, 1992).

[2] L. Onsager, Ann. N.Y. Acad. Sci. 51, 627 (1949).

[3] P. J. Flory, Proc. R. Soc. London, Ser. A 234, 73 (1956).

[4] D. Frenkel, H. N. W. Lekkerkerker, and A. Stroobants, Nature (London) 332, 822 (1988).

[5] J. Tang and S. Fraden, Liq. Cryst. 19, 459 (1995).

[6] T. Sato and A. Teramoto, Adv. Polym. Sci. 126, 85 (1996).

[7] M. P. B. van Bruggen, F. M. van der Kooij, and H. N. W. Lekkerkerker, J. Phys.: Condens. Matter 8, 9451 (1996).

[8] P. J. Flory and A. Abe, Macromolecules 11, 1119 (1978).

[9] T. Itou and A. Teramoto, Macromolecules 17, 1419 (1984).

[10] T. Itou and A. Teramoto, Polym. J. (Tokyo, Jpn.) 16, 779 (1984).

[11] P. A. Buining and H. N. W. Lekkerkerker, J. Phys. Chem. 97, 11510 (1993).

[12] G. Nouensis, S. Kumar, S. Pfieffer, R. Shashidhar, and C. W. Garland, Phys. Rev. Lett. 73, 565 (1994).

[13] F. M. van der Kooij and H. N. W. Lekkerkerker, Phys. Rev. Lett. 84, 781 (2000).

[14] F. M. van der Kooij and H. N. W. Lekkerkerker, Langmuir 16, 10144 (2000).

[15] K. R. Purdy, S. Varga, A. Galindo, G. Jackson, and S. Fraden, Phys. Rev. Lett. 94, 057801 (2005).

[16] E. Grelet and S. Fraden, Phys. Rev. Lett. 90, 198302 (2003).

[17] P. J. Camp and M. P. Allen, Physica A 229, 410 (1996); P. J. Camp, M. P. Allen, P. G. Bolhuis, and D. Frenkel, J. Chem. Phys. 106, 9270 (1997).

[18] A. Galindo, G. Jackson, and D. Photinos, Chem. Phys. Lett. 325, 631 (2000).

[19] A. Galindo, A. J. Haslam, S. Varga, G. Jackson, A. G. Vanakaras, D. J. Photinos, and D. A. Dunmar, J. Chem. Phys. 119, 5216 (2003).

[20] X. Zhou, H. Chen, and M. Iwamoto, J. Chem. Phys. 120, 1832 (2004).

[21] P. G. Bolhuis, A. Stroobants, D. Frenkel, and H. N. W. Lekkerkerker, J. Chem. Phys. 107, 1551 (1997).

[22] M. Dijkstra and R. van Roij, Phys. Rev. E 56, 5594 (1997).

[23] M. Birshtein, B. I. Kogelov, and V. A. Pryamitsin, Vysokomol. Soedin., Ser. A 30, 348 (1988); [Polym. Sci. U.S.S.R. 30, 316 (1988)].

[24] H. N. W. Lekkerkerker, Ph. Coulon, R. Van Der Haegen, and R. Deblieck, J. Chem. Phys. 80, 3427 (1984).

[25] G. J. Vroege and H. N. W. Lekkerkerker, J. Phys. Chem. 97, 3601 (1993).
[26] R. van Roij and B. Mulder, J. Chem. Phys. 105, 11237 (1996); R. van Roij and B. Mulder, J. Chem. Phys. 109, 1584(E) (1998).

[27] S. Varga and I. Szalai, Phys. Chem. Chem. Phys. 2, 1955 (2000).

[28] P. C. Hemmer, Mol. Phys. 96, 1153 (1999).

[29] R. P. Sear and B. M. Mulder, J. Chem. Phys. 105, 7727 (1996).

[30] R. van Roij and B. Mulder, Phys. Rev. E 54, 6430 (1996).

[31] R. van Roij, B. M. Mulder, and M. Dijkstra, Physica A 261, 347 (1998).

[32] A. Speranza and P. Sollich, J. Chem. Phys. 117, 5421 (2002).

[33] S. Varga, A. Galindo, and G. Jackson, Mol. Phys. 101, 817 (2003).

[34] P. Palffy-Muhoray, A. J. Berlinsky, J. R. de Bruyn, and D. A. Dunmur, Phys. Lett. 104, 159 (1984).

[35] P. Sollich, J. Chem. Phys. 122, 214911 (2005).

[36] A. Speranza and P. Sollich, e-print cond-mat/0208401.

[37] S. DuBois and A. Perera, J. Chem. Phys. 116, 6354 (2002).

[38] A. Perera, J. Mol. Liq. 109, 73 (2004).

[39] H. H. Wensink, G. J. Vroege, and H. N. W. Lekkerkerker, J. Phys. Chem. B 105, 10610 (2001).

[40] S. Varga, A. Galindo, and G. Jackson, J. Chem. Phys. 117, 10412 (2002).

[41] S. Varga, A. Galindo, and G. Jackson, Phys. Rev. E 66, 011707 (2002).

[42] J. S. Rowlinson and F. L. Swinton, Liquids and Liquid Mixtures, 3rd ed. (Butterworth Scientific, London, 1982); H. B. Callen, Thermodynamics (Wiley, New York, 1960).

[43] J. D. Parsons, Phys. Rev. A 19, 1225 (1979).

[44] S. D. Lee, J. Chem. Phys. 87, 4972 (1987).

[45] T. Odijk and H. N. W. Lekkerkerker, J. Phys. Chem. 89, 2090 (1985).

[46] J. P. Straley, Mol. Cryst. Liq. Cryst. 24, 7 (1973).

[47] G. J. Vroege and H. N. W. Lekkerkerker, Rep. Prog. Phys. 55, 1241 (1992).

[48] A. Poniewierski, Phys. Rev. A 45, 5605 (1992).

[49] S. C. McGrother, D. C. Williamson, and G. Jackson, J. Chem. Phys. 104, 6755 (1996).

[50] P. J. Camp, C. P. Mason, M. P. Allen, A. A. Khare, and D. A. Kofke, J. Chem. Phys. 105, 2837 (1996).

[51] M. Franco-Melgar, S. Varga, and G. Jackson (unpublished).

[52] P. Bolhuis and D. Frenkel, J. Chem. Phys. 106, 666 (1997).

[53] J. P Hansen and I. R. McDonald, Theory of Simple Liquids, 2nd ed. (Academic Press, London, 1986). 